Article

\title{
TDAE Strategy in the Benzoxazolone Series: Synthesis and Reactivity of a New Benzoxazolinonic Anion
}

\author{
Aïda R. Nadji-Boukrouche ${ }^{1,2,3}$, Omar Khoumeri ${ }^{3}$, Thierry Terme ${ }^{3}$, Messaoud Liacha ${ }^{2}$ \\ and Patrice Vanelle ${ }^{3, *}$
}

1 Département de Génie des Procédés, Université 8 mai 1945 Guelma, BP 401, Guelma 24000, Algeria; E-Mail: nadji_aida@yahoo.fr

2 Laboratoire de Synthèse et de Biocatalyse Organique (LSBO), Faculté des Sciences, Université Badji Mokhtar-Annaba, BP 12 El-Hadjar, Annaba 23000, Algeria;

E-Mail: m_liacha@yahoo.fr

3 Aix-Marseille Université, CNRS, Institut de Chimie Radicalaire ICR, UMR 7273, Laboratoire de Pharmaco-Chimie Radicalaire, Marseille 13385, France;

E-Mails: omar.khoumeri@univ-amu.fr (O.K.); thierry.terme@univ-amu.fr (T.T.)

* Author to whom correspondence should be addressed; E-Mail: patrice.vanelle@univ-amu.fr; Tel.: +33-4-9183-5580; Fax: +33-4-9179-4677.

Academic Editor: Derek J. McPhee

Received: 11 December 2014 / Accepted: 8 January 2015 / Published: 14 January 2015

\begin{abstract}
We describe an original pathway to produce new 5-substituted 3-methyl-6-nitrobenzoxazolones by the reaction of aromatic carbonyl and $\alpha$-carbonyl ester derivatives with a benzoxazolinonic anion formed exclusively via the TDAE strategy.
\end{abstract}

Keywords: TDAE; benzoxazolone; benzoxazolinonic anion; benzylic alcohols; oxiranes

\section{Introduction}

Many benzoxazolinone derivatives have been described in therapeutics as possessing a wide variety of pharmacological activities [1-10]. Indeed, the clinical applications of this template are very broad, and range from analgesic anti-inflammatory compounds to antipsychotic and neuroprotective anticonvulsant compounds [11]. Several potentially useful drugs and pharmacological tools based on these pharmacophores have been developed in recent years [12-16]. 
Tetrakis(dimethylamino)ethylene (TDAE) is a reducing agent which reacts with halogenated derivatives to generate an anion under mild conditions via two sequential transfers of one electron [17-19]. Through this strategy, we have developed many reactions between nitrobenzylic substrates and a series of electrophiles such as aldehydes, ketones, $\alpha$-ketoesters, $\alpha$-ketolactams and ketomalonates leading to corresponding alcohol adducts [20-23]. This reactivity was recently extended using original heterocyclic carbaldehydes as electrophiles. The reactions led to the expected products, while at the same time bringing to light a new and original reactivity and enabling us to define some limitations of this strategy [24]. Moreover, we reported the reactions of dihalo- and trihalomethyl heterocyclic derivatives with aromatic aldehydes in the presence of TDAE, providing a mixture of cis/trans isomers of oxiranes and $\alpha$-haloketone derivatives, respectively $[25,26]$. In the same context, the expected alcohols and oxiranes were obtained in good yields in the quinonic series [27].

In continuation of our research program centered on the design and synthesis of novel bioactive molecules [28-32], we report herein the preparation of 5-(bromomethyl)-3-methyl-6-nitrobenzoxazolone (2) and 5-(dibromomethyl)-3-methyl-6-nitrobenzoxazolone (3) and the study of their reactivity with various aromatic carbonyl and $\alpha$-carbonyl ester derivatives using the TDAE methodology.

\section{Results and Discussion}

\subsection{Synthesis of Mono and Dibromide Substrates}

We prepared 5-(bromomethyl)-3-methyl-6-nitrobenzoxazolone (2) and 5-(dibromomethyl)-3methyl-6-nitrobenzoxazolone (3) [33] in four and five steps, respectively. The condensation of 2-amino-4-methylphenol with urea was inspired by a previously described method [34,35]. After methylation using dimethyl sulfate, the nitration of the obtained 3,5-dimethylbenzoxazolone by action of a mixture of nitric and sulfuric acids afforded 3,5-dimethyl-6-nitrobenzoxazolone (1) in 88\% yield.

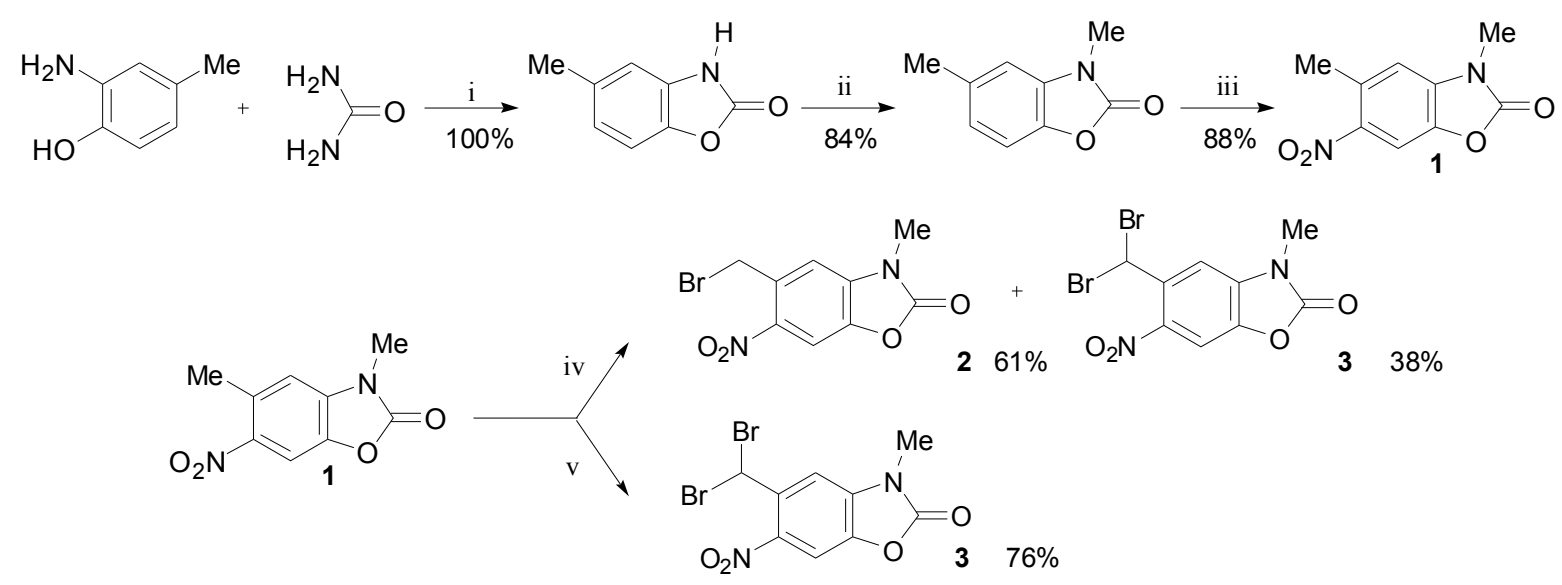

i) DMF, $145-150^{\circ} \mathrm{C}, 3$ h30. ii) DMS, r.t., 3 h. iii) $\mathrm{HNO}_{3}$ (1 eq.), $\mathrm{H}_{2} \mathrm{SO}_{4}, 0{ }^{\circ} \mathrm{C}, 2 \mathrm{~h}$. iv) NBS (2 eq.), hv, benzoyl peroxide, $\mathrm{CCl}_{4}, 80^{\circ} \mathrm{C}, 8.5 \mathrm{~h}$ v) NBS (6 eq.), hv, benzoyl peroxide, $\mathrm{CCl}_{4}, 80^{\circ} \mathrm{C}, 10 \mathrm{~h}$.

Scheme 1. Synthesis of 5-(bromomethyl)-3-methyl-6-nitrobenzoxazolone (2) and 5-(dibromomethyl)-3-methyl-6-nitrobenzoxazolone (3).

The bromination of 1 with 2 equivalents of $\mathrm{N}$-bromosuccinimide in refluxing $\mathrm{CCl}_{4}$ for $8.5 \mathrm{~h}$ gave 5-(bromomethyl)-3-methyl-6-nitrobenzoxazolone (2) in 61\% yield, accompanied by 5-(dibromomethyl)- 
3-methyl-6-nitrobenzoxazolone (3) in 38\% yield. However, the preparation of this latter compound was optimized (76\%) using 6 equivalents of $\mathrm{N}$-bromosuccinimide in refluxing $\mathrm{CCl}_{4}$ for $10 \mathrm{~h}$ (Scheme 1).

\subsection{TDAE Reactivity of 5-(Bromomethyl)-3-methyl-6-nitrobenzoxazolone}

The reaction of 5-(bromomethyl)-3-methyl-6-nitrobenzoxazolone (2) with 3 equivalents of various aromatic carbonyl and $\alpha$-carbonyl ester derivatives $4 \mathbf{a}-\mathbf{j}$ in the presence of TDAE at $-20{ }^{\circ} \mathrm{C}$ for $1 \mathrm{~h}$, followed by $2 \mathrm{~h}$ at room temperature (r.t.) led to the corresponding alcohol derivatives $\mathbf{5 a}-\mathbf{j}$ in moderate to good yields $(31 \%-72 \%)$ as shown in Table 1 and Scheme 2.

Table 1. Reaction of bromide 2 with aromatic carbonyl and $\alpha$-carbonyl ester derivatives using TDAE ${ }^{\mathrm{a}}$.

\begin{tabular}{|c|c|c|c|c|c|}
\hline Entry $^{a}$ & Aromatic Carbonyl & $R_{1}$ & $R_{2}$ & Product Number & Yield $(\%)^{b}$ \\
\hline 1 & 4-Nitrobenzaldehyde & $4-\mathrm{NO}_{2}-\mathrm{C}_{6} \mathrm{H}_{4}$ & $\mathrm{H}$ & $5 \mathbf{5 a}$ & 52 \\
\hline 2 & 4-Bromobenzaldehyde & $4-\mathrm{Br}_{-} \mathrm{C}_{6} \mathrm{H}_{4}$ & $\mathrm{H}$ & $5 \mathbf{b}$ & 49 \\
\hline 3 & 4-Cyanobenzaldehyde & $4-\mathrm{CN}-\mathrm{C}_{6} \mathrm{H}_{4}$ & $\mathrm{H}$ & $5 c$ & 31 \\
\hline 4 & 2-Nitrobenzaldehyde & $2-\mathrm{NO}_{2}-\mathrm{C}_{6} \mathrm{H}_{4}$ & $\mathrm{H}$ & 5d & 44 \\
\hline 5 & 2-Bromobenzaldehyde & $2-\mathrm{Br}-\mathrm{C}_{6} \mathrm{H}_{4}$ & $\mathrm{H}$ & $5 e$ & 49 \\
\hline 6 & 3-Bromobenzaldehyde & $3-\mathrm{Br}-\mathrm{C}_{6} \mathrm{H}_{4}$ & $\mathrm{H}$ & $5 f$ & 43 \\
\hline 7 & Ethyl glyoxylate & $\mathrm{CO}_{2} \mathrm{C}_{2} \mathrm{H}_{5}$ & $\mathrm{H}$ & $5 \mathrm{~g}$ & 72 \\
\hline 8 & Diethyl ketomalonate & $\mathrm{CO}_{2} \mathrm{C}_{2} \mathrm{H}_{5}$ & $\mathrm{CO}_{2} \mathrm{C}_{2} \mathrm{H}_{5}$ & $5 \mathrm{~h}$ & 62 \\
\hline
\end{tabular}

Notes: a All the reactions were performed using 3 equivalents of aromatic carbonyl $\mathbf{4 a}-\mathbf{h}, 1$ equivalent of bromide 2 and 1 equivalent of TDAE in anhydrous DMF stirred at $-20{ }^{\circ} \mathrm{C}$ for $1 \mathrm{~h}$ and then warmed to $\mathrm{rt}$ for $2 \mathrm{~h}$; b $\%$ Yield relative to bromide 2.

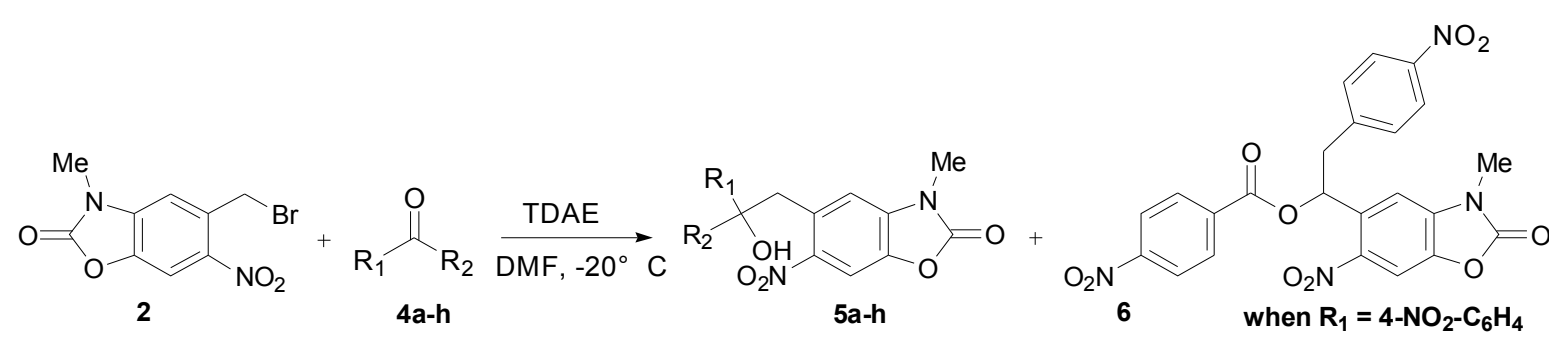

Scheme 2. TDAE reactivity of 5-(bromomethyl)-3-methyl-6-nitrobenzoxazolone (2) with aromatic carbonyl and some $\alpha$-keto-ester derivatives $\mathbf{4 a - h}$.

The reaction of substrate $\mathbf{2}$ with the aromatic aldehydes $\mathbf{4 a}-\mathbf{f}$ under TDAE-initiated conditions furnished the expected alcohols $\mathbf{5 a}-\mathbf{f}$ in moderate to good yields. The best yield (52\%) was obtained with $p$-nitrobenzaldehyde (4a). Unexpectedly, o,p-bromobenzaldehyde $(\mathbf{4 e}, \mathbf{4 b})$ gave the same yield (49\%), while $o$-nitrobenzaldehyde (4d) and $m$-bromobenzaldehyde (4f) gave approximately the same yield (44\% and $43 \%$, respectively). Notably, with $p$-nitrobenzaldehyde (4a) we observed $23 \%$ of the ester 6. According to a recent mechanistic study [36], the formation of the unexpected ester derivative 6 may be explained by an electron transfer in a primary step between 4-nitrobenzaldehyde (4a) as acceptor and TDAE as donor.

p-Cyanobenzaldehyde (4c) produced a moderate yield $(31 \%)$. The formation of these alcohol derivatives may be explained by nucleophilic addition of benzazolinonic carbanions formed by the 
action of TDAE with 5-(bromomethyl)-3-methyl-6-nitrobenzoxazolone (2) on the carbonyl group of the corresponding aldehyde. In summary, the difference in yields does not appear to be totally explained by electronic effects: the halogen groups furnished approximately the same yields in either position. With nitrobenzaldehydes, steric hindrance could explain the difference between $o$ - and $p$-nitrobenzaldehyde yields (44\% versus $52 \%$ ).

It is important to note that in the reactions of substrate $\mathbf{2}$ with the electrophiles $\mathbf{4 b}-\mathbf{f}$, we observed the unavoidable formation of the reduction product 1 [37]. Extending the reaction times to $8 \mathrm{~h}$ at ambient temperature increases its percentage, but decreases the yield of alcohol. On the other hand, after $4 \mathrm{~h}$ of reaction, the percentage of reduction product decreases at the same time as that of the alcohol: in this case we also observed traces of the dimerization of substrate 2 .

Moreover, after the reaction with aromatic aldehydes, we investigated the reaction of 2 with $\alpha$-keto-ester derivatives such as ethyl glyoxylate (4g), diethyl ketomalonate (4h), acenaphtenedione (4i) and 1-methylisatin $(\mathbf{4 j})$ in the presence of TDAE. The reactions with these electrophiles furnished the corresponding hydroxyl derivatives $\mathbf{5 i - \mathbf { j }}$ in good yields (59\%-63\%), as shown in Table 1 and Scheme 3.

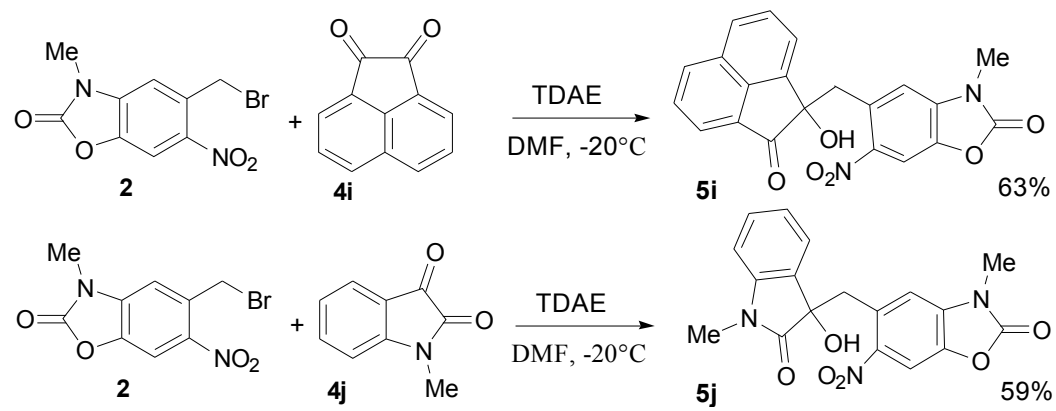

Scheme 3. TDAE reactivity of the 5-(bromomethyl)-3-methyl-6-nitrobenzoxazolone (2) and $\alpha$-diketone and $\alpha$-ketolactam derivatives $4 \mathbf{i}-\mathbf{j}$.

\subsection{TDAE Reactivity of 5-(Dibromomethyl)-3-methyl-6-nitrobenzoxazolone (3)}

The optimized protocol of the dibromomethyl derivative 3, was defined with 3 equivalents of aromatic carbonyls $\mathbf{4 a}-\mathbf{h}, 1$ equivalent of 5-(dibromomethyl)-3-methyl-6-nitrobenzoxazolone (3) and 1.5 equivalents of TDAE in anhydrous DMF, for $1 \mathrm{~h}$ at $-20^{\circ} \mathrm{C}$ followed by $2 \mathrm{~h}$ at r.t. The reactions led to a mixture of cis/trans isomers of the corresponding oxiranes $7 \mathbf{a}-\mathbf{h}$ in moderate to good yields as reported in Table 2 (Scheme 4). The formation of these oxiranes may be explained by nucleophilic addition of a $\alpha$-bromocarbanion, formed by the action of TDAE with 5-(dibromomethyl)-3-methyl-6nitrobenzoxazolone (3), on the carbonyl group of aldehydes $\mathbf{4 a}-\mathbf{h}$ followed by an intramolecular nucleophilic substitution [26].

In the case of the nitroaromatic aldehydes, steric hindrance could explain the yield difference between $o$ - and $p$-nitrobenzaldehyde (46\% and 63\%). However, this effect disappears in the $o$-bromo-substituted aldehyde which gave $64 \%$ of the corresponding oxirane, the $p$ - and $m$ - substituted aldehydes with 55 and $48 \%$ yields respectively. $p$-Cyanobenzaldehyde gave the expected oxirane in good yield $(72 \%)$.

Under the same experimental conditions, we studied the reaction of derivative $\mathbf{3}$ with $\alpha$-keto-ester derivatives $\mathbf{4 g}-\mathbf{h}$ as reported in Table 2 (Scheme 4). Only the trans isomers of the oxiranes $\mathbf{7 g}$ and $\mathbf{7 h}$ were obtained in $26 \%$ and $37 \%$ yields, respectively, with ethyl glyoxylate (4g) and diethyl ketomalonate 
(4h). Otherwise, acenaphtenedione (4i) and methyl isatin (4j) furnished mixtures of like/unlike original stereoisomers $7 \mathbf{i}$ and $\mathbf{7 j}$, respectively, in good yields (Scheme 5). The diastereoisomers were separable, and their configuration was identified by NMR-analysis from the $\gamma$-left effect, as previously described [26,38].

Table 2. Reaction of dibromide 3 with aromatic carbonyl and $\alpha$-carbonyl ester derivatives using TDAE ${ }^{\mathrm{a}}$.

\begin{tabular}{|c|c|c|c|c|c|c|}
\hline Entry $^{a}$ & Aromatic Carbonyl & $R_{1}$ & $\boldsymbol{R}_{2}$ & Oxirane & Cis/Trans Isomers \% ${ }^{\mathrm{b}}$ & Yield (\%) ${ }^{\mathrm{c}}$ \\
\hline 1 & 4-Nitrobenzaldehyde & $4-\mathrm{NO}_{2}-\mathrm{C}_{6} \mathrm{H}_{4}$ & $\mathrm{H}$ & $7 a$ & $15 / 85$ & 63 \\
\hline 2 & 4-Bromobenzaldehyde & $4-\mathrm{Br}-\mathrm{C}_{6} \mathrm{H}_{4}$ & $\mathrm{H}$ & $7 b$ & $7 / 93$ & 55 \\
\hline 3 & 4-Cyanobenzaldehyde & $4-\mathrm{CN}-\mathrm{C}_{6} \mathrm{H}_{4}$ & $\mathrm{H}$ & $7 \mathrm{c}$ & $15 / 85$ & 72 \\
\hline 4 & 2-Nitrobenzaldehyde & $2-\mathrm{NO}_{2}-\mathrm{C}_{6} \mathrm{H}_{4}$ & $\mathrm{H}$ & 7d & $32 / 68$ & 46 \\
\hline 5 & 2-Bromobenzaldehyde & $2-\mathrm{Br}_{-} \mathrm{C}_{6} \mathrm{H}_{4}$ & $\mathrm{H}$ & $7 e$ & $19 / 81$ & 64 \\
\hline 6 & 3-Bromobenzaldehyde & $3-\mathrm{Br}-\mathrm{C}_{6} \mathrm{H}_{4}$ & $\mathrm{H}$ & $7 f$ & $7 / 93$ & 48 \\
\hline 7 & Ethyl glyoxylate & $\mathrm{CO}_{2} \mathrm{C}_{2} \mathrm{H}_{5}$ & $\mathrm{H}$ & $7 \mathrm{~g}$ & $0 / 100$ & 26 \\
\hline 8 & Diethyl ketomalonate & $\mathrm{CO}_{2} \mathrm{C}_{2} \mathrm{H}_{5}$ & $\mathrm{CO}_{2} \mathrm{C}_{2} \mathrm{H}_{5}$ & $7 \mathrm{~h}$ & $0 / 100$ & 37 \\
\hline
\end{tabular}

Notes: ${ }^{\text {a }}$ All the reactions were performed using 3 equivalents of aromatic carbonyl $\mathbf{4 a}-\mathbf{h}, 1$ equivalent of dibromide 3 and 1.5 equivalent of TDAE in anhydrous DMF stirred at $-20{ }^{\circ} \mathrm{C}$ for $1 \mathrm{~h}$ and then warmed to r.t for $2 \mathrm{~h}$; ${ }^{\mathrm{b}} \%$ isomers determined on ${ }^{1} \mathrm{H}-\mathrm{NMR}$ measurements from the crude product; ${ }^{\mathrm{c}} \%$ yield relative to dibromide 3.

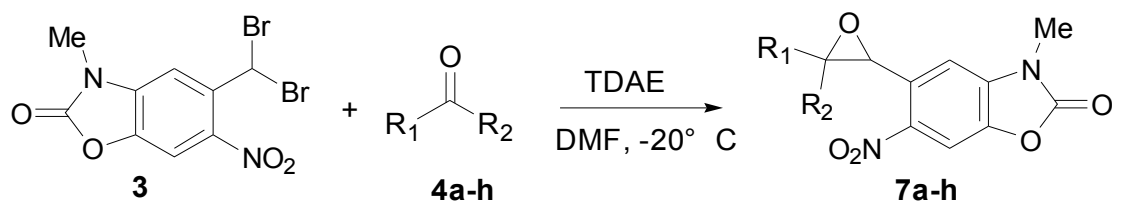

Scheme 4. TDAE reactivity of 5-(dibromomethyl)-3-methyl-6-nitrobenzoxazolone (3) with aromatic carbonyl and some $\alpha$-keto-ester derivatives $\mathbf{4 a}-\mathbf{h}$.
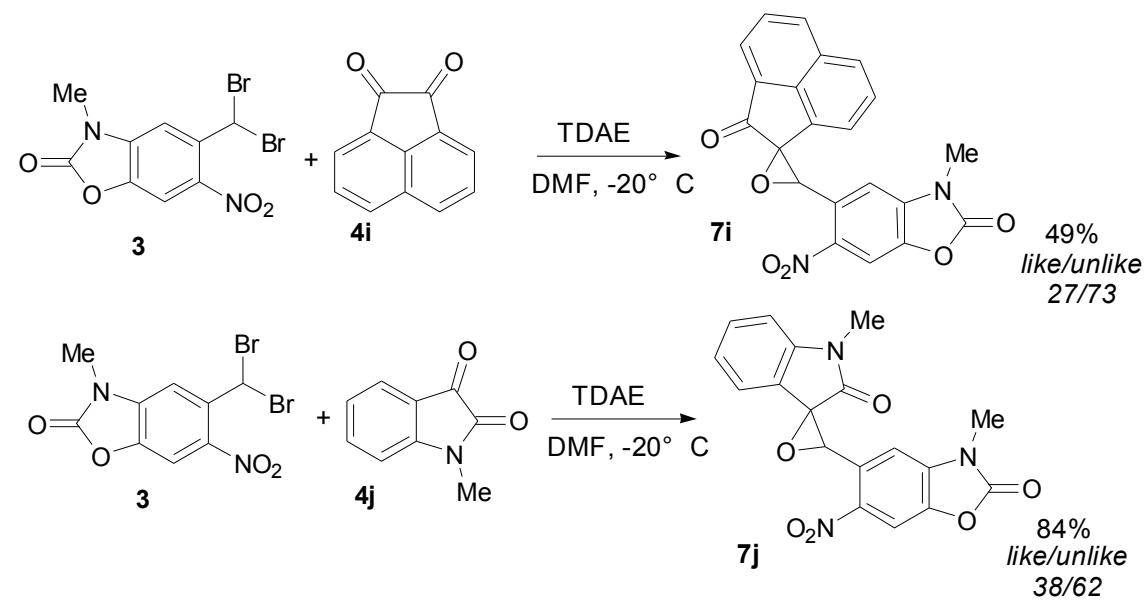

Scheme 5. TDAE reactivity of 5-(dibromomethyl)-3-methyl-6-nitrobenzoxazolone (3) and $\alpha$-keto-ester derivatives $\mathbf{4} \mathbf{i}-\mathbf{j}$.

The relative cis/trans percentages of oxirane isomers reported in Table 2 showed that the stereoselectivity of these reactions is not only sensitive to steric hindrance, but it also depends on the nature of the electrophile substituents. The reactions with bromo-substituted aldehydes in either position were more 
selective than with nitro-substituted aldehydes. The same percentages of cis/trans isomers were previously reported with $p$-nitro- and cyanobenzaldehyde. However, the reactions with ethyl glyoxylate and diethyl ketomalonate were the most selective. Moreover, stereoselectivity was recorded in the mixtures of like/unlike original stereoisomers with methyl isatin and acenaphtenedione.

\section{Experimental Section}

\subsection{General Information}

Melting points were determined on a Buchi capillary melting point apparatus and are uncorrected. Elemental analyses were performed by the Centre de Microanalyses of the University of Aix-Marseille. Both ${ }^{1} \mathrm{H}-(200 \mathrm{MHz})$ and ${ }^{13} \mathrm{C}-\mathrm{NMR}(50 \mathrm{MHz})$ spectra were determined on a Bruker AC 200 spectrometer. The ${ }^{1} \mathrm{H}$ chemical shifts are reported as parts per million downfield from tetramethylsilane $\left(\mathrm{Me}_{4} \mathrm{Si}\right)$, and the ${ }^{13} \mathrm{C}$ chemical shifts were referenced to the solvent peaks: $\mathrm{CDCl}_{3}$ (76.9 ppm) or $\mathrm{Me}_{2} \mathrm{SO}-d_{6}(39.6 \mathrm{ppm}$ ). Absorptions are reported using the following notation: s, singlet; $d$, doublet; $t$, triplet; q, quartet; $\mathrm{m}$, a more complex multiplet or overlapping multiplets. The following adsorbents were used for column chromatography: silica gel 60 (Merck, Darmstadt, Germany, particle size 0.063-0.200 mm, 70-230 mesh ASTM). TLC was performed on $5 \mathrm{~cm} \times 10 \mathrm{~cm}$ aluminium plates coated with silica gel $60 \mathrm{~F}-254$ (Merck) in an appropriate solvent. 3,5-Dimethyl-6-nitrobenzoxazolone (1) was synthesized in three steps: condensation of 2-amino-4-methylphenol with urea [34], methylation using dimethyl sulfate and nitration by action of a mixture of nitric and sulfuric acids.

\subsection{Synthesis of Substrates 1-3}

3,5-Dimethyl-6-nitrobenzo[d]oxazol-2(3H)-one (1): yellow solid; mp $159{ }^{\circ} \mathrm{C}$ (EtOH); ${ }^{1} \mathrm{H}-\mathrm{NMR}$ $\left(\mathrm{CDCl}_{3}\right): \delta 2.70\left(\mathrm{~s}, 3 \mathrm{H}, \mathrm{CH}_{3}\right), 3.45\left(\mathrm{~s}, 3 \mathrm{H}, \mathrm{NCH}_{3}\right), 6.87(\mathrm{~s}, 1 \mathrm{H}, \mathrm{CH}), 7.95(\mathrm{~s}, 1 \mathrm{H}, \mathrm{CH}) .{ }^{13} \mathrm{C}-\mathrm{NMR}\left(\mathrm{CDCl}_{3}\right)$ : $\delta 21.6\left(\mathrm{CH}_{3}\right), 28.5\left(\mathrm{NCH}_{3}\right), 107.3(\mathrm{CH}), 110.7(\mathrm{CH}), 132.2(\mathrm{C}), 135.7(\mathrm{C}), 140.2(\mathrm{C}), 143.5\left(\mathrm{CNO}_{2}\right)$, 154.3 (CO). Anal. Calcld. for $\mathrm{C}_{9} \mathrm{H}_{8} \mathrm{~N}_{2} \mathrm{O}_{4}$ (208.17): C, 51.93; H, 3.87; N; 13.46. Found: C, 52.34; H, 3.95; $\mathrm{N}, 13.40$.

5-(Bromomethyl)-3-methyl-6-nitrobenzo[d]oxazol-2(3H)-one (2) and 5-(dibromomethyl)-3-methyl6-nitrobenzo[ $d]$ oxazol-2(3H)-one (3) were prepared according to a previously described method [27].

5-(Bromomethyl)-3-methyl-6-nitrobenzo[d] oxazol-2(3H)-one (2): yellow solid (EtOH); mp $120{ }^{\circ} \mathrm{C}$; ${ }^{1} \mathrm{H}-\mathrm{NMR}\left(\mathrm{CDCl}_{3}\right): \delta 3.49\left(\mathrm{~s}, 3 \mathrm{H}, \mathrm{CH}_{3}\right), 4.91\left(\mathrm{~s}, 2 \mathrm{H}, \mathrm{CH}_{2} \mathrm{Br}\right), 7.14(\mathrm{~s}, 1 \mathrm{H}, \mathrm{CH}), 7.99(\mathrm{~s}, 1 \mathrm{H}, \mathrm{CH})$. ${ }^{13} \mathrm{C}-\mathrm{NMR}\left(\mathrm{CDCl}_{3}\right): \delta 28.7\left(\mathrm{NCH}_{3}\right), 29.3\left(\mathrm{CH}_{2} \mathrm{Br}\right), 108.0(\mathrm{CH}), 110.6(\mathrm{CH}), 131.1(\mathrm{C}), 136.1(\mathrm{C}), 141.7$ (C), $142.6\left(\mathrm{CNO}_{2}\right), 153.9$ (CO). Anal. Calcld for $\mathrm{C}_{9} \mathrm{H}_{7} \mathrm{BrN}_{2} \mathrm{O}_{4}$ (287.07): C, 37.66; H, 2.46; N; 9.76. Found: C, 38.48; H, 2.58; N, 9.88.

5-(Dibromomethyl)-3-methyl-6-nitrobenzo[d]oxazol-2(3H)-one (3): yellow solid (EtOH); mp $134{ }^{\circ} \mathrm{C}$; ${ }^{1} \mathrm{H}-\mathrm{NMR}\left(\mathrm{CDCl}_{3}\right): \delta 3.55\left(\mathrm{~s}, 3 \mathrm{H}, \mathrm{NCH}_{3}\right), 7.55\left(\mathrm{~s}, 1 \mathrm{H}, \mathrm{CHBr}_{2}\right), 7.75(\mathrm{~s}, 1 \mathrm{H}, \mathrm{CH}), 7.78(\mathrm{~s}, 1 \mathrm{H}, \mathrm{CH})$. ${ }^{13} \mathrm{C}-\mathrm{NMR}\left(\mathrm{CDCl}_{3}\right): \delta 29.0\left(\mathrm{NCH}_{3}\right), 34.4\left(\mathrm{CHBr}_{2}\right), 106.2(\mathrm{CH}), 110.7(\mathrm{CH}), 134.3(\mathrm{C}), 136.5(\mathrm{C}), 139.0$ (C), $142.0\left(\mathrm{CNO}_{2}\right), 153.6$ (CO). Anal. Calcld for $\mathrm{C}_{9} \mathrm{H}_{7} \mathrm{BrN}_{2} \mathrm{O}_{4}$ (365.96): C, 29.54; H, 1.65; N; 7.65. Found: C, 29.59; H, 1.67; N, 7.69. 
3.3. General Procedure for the Reaction of $\mathbf{2}$ and Aromatic Carbonyl Derivatives $\mathbf{4 a}-\mathbf{f}, \alpha$-Carbonyl Ester 4g, Ketomalonate 4h, Acenaphtenedione $\mathbf{4 i}$ and Ketolactam $\mathbf{4 j}$ Using TDAE

A solution of $2(0.5,1.74 \mathrm{mmol})$ in anhydrous DMF $(10 \mathrm{~mL})$ and the corresponding carbonyl derivative $4 \mathbf{a}-\mathbf{j}$ (5.22 mmol, 3 equivalents) were placed under nitrogen at $-20{ }^{\circ} \mathrm{C}$ in a two-necked flask equipped with a silica-gel drying tube and a nitrogen inlet. The solution was stirred and maintained at this temperature for $30 \mathrm{~min}$ and then the TDAE $(0.41 \mathrm{~mL}, 1.74 \mathrm{mmol}, 1$ equivalent $)$ was added dropwise via a syringe. A red color immediately developed with the formation of a fine white precipitate. The solution was vigorously stirred at $-20{ }^{\circ} \mathrm{C}$ for $1 \mathrm{~h}$ and then warmed to r.t. for $2 \mathrm{~h}$. After this time TLC analysis (dichloromethane) clearly showed that $\mathbf{2}$ was totally consumed. The orange-red turbid solution was filtered (to remove the octamethyloxamidinium dibromide) and hydrolyzed with $80 \mathrm{~mL}$ of $\mathrm{H}_{2} \mathrm{O}$. The aqueous solution was extracted with toluene $(3 \times 40 \mathrm{~mL})$, the combined organic layers washed with $\mathrm{H}_{2} \mathrm{O}(3 \times 40 \mathrm{~mL})$ and dried over $\mathrm{MgSO}_{4}$. Evaporation of the solvent left an orange viscous liquid as crude product. Purification by silica gel chromatography and recrystallization in ethyl alcohol gave the corresponding products.

5-(2-Hydroxy-2-(4-nitrophenyl)ethyl)-3-methyl-6-nitrobenzo[d]oxazol-2(3H)-one (5a): Brown solid; mp $233{ }^{\circ} \mathrm{C}$; ${ }^{1} \mathrm{H}-\mathrm{NMR}$ (DMSO- $\left.d_{6}\right): \delta 3.37$ (s, 3H, $\left.\mathrm{NCH}_{3}\right), 3.17-3.33(\mathrm{~m}, 2 \mathrm{H}, 2 \times \mathrm{CH}), 4.92-5.01(\mathrm{~m}, 1 \mathrm{H}$, $1 \mathrm{H}, \mathrm{CH}), 5.67$ (bs, 1H, OH), $7.33(\mathrm{~s}, 1 \mathrm{H}, \mathrm{CH}), 7.61(\mathrm{~d}, J=8,5 \mathrm{~Hz}, 2 \mathrm{H}, 2 \times \mathrm{CH}), 8.00(\mathrm{~s}, 1 \mathrm{H}, \mathrm{CH}), 8.21$ $(\mathrm{d}, J=8.5 \mathrm{~Hz}, 2 \mathrm{H}, 2 \times \mathrm{CH}) .{ }^{13} \mathrm{C}-\mathrm{NMR}\left(\mathrm{DMSO}-d_{6}\right): \delta 28.9\left(\mathrm{NCH}_{3}\right), 42.2\left(\mathrm{CH}_{2}\right), 72.1(\mathrm{CH}), 106.7(\mathrm{CH})$, $112.5(\mathrm{CH}), 123.6(2 \times \mathrm{CH}), 127.0(2 \times \mathrm{CH}), 131.2(\mathrm{C}), 135.8(\mathrm{C}), 140.2(\mathrm{C}), 144.1(\mathrm{C}), 146.7(\mathrm{C}), 153.2$ (C), 154.3 (CO). HRMS (EI): calcd for $\mathrm{C}_{16} \mathrm{H}_{13} \mathrm{~N}_{3} \mathrm{O}_{7}\left(\mathrm{M}^{+}\right)$337.1092, found 337.1092.

5-(2-(4-Bromophenyl)-2-hydroxyethyl)-3-methyl-6-nitrobenzo[d]oxazol-2(3H)-one (5b): Brown solid; mp $213{ }^{\circ} \mathrm{C} ;{ }^{1} \mathrm{H}-\mathrm{NMR}\left(\mathrm{CDCl}_{3}\right): \delta 2.13(\mathrm{~d}, J=3.2 \mathrm{~Hz}, 1 \mathrm{H}, \mathrm{OH}), 3.13(\mathrm{dd}, J=13.7 \mathrm{~Hz}, J=9.1 \mathrm{~Hz}, 1 \mathrm{H}$, $\mathrm{CH}), 3.43\left(\mathrm{~s}, 3 \mathrm{H}, \mathrm{NCH}_{3}\right), 3.50(\mathrm{dd}, J=13.7 \mathrm{~Hz}, J=3.7 \mathrm{~Hz}, 1 \mathrm{H}, \mathrm{CH}), 5.06(\mathrm{dd}, J=9.1 \mathrm{~Hz}, J=3.7 \mathrm{~Hz}, 1 \mathrm{H}$, $\mathrm{CH}), 6.85(\mathrm{~s}, 1 \mathrm{H}, \mathrm{CH}), 7.33(\mathrm{~d}, J=8.4 \mathrm{~Hz}, 2 \mathrm{H}, 2 \times \mathrm{CH}), 7.52(\mathrm{~d}, J=8.4 \mathrm{~Hz}, 2 \mathrm{H}, 2 \times \mathrm{CH}), 7.94(\mathrm{~s}, 1 \mathrm{H}$, $\mathrm{CH}) .{ }^{13} \mathrm{C}-\mathrm{NMR}\left(\mathrm{CDCl}_{3}\right): \delta 28.6\left(\mathrm{NCH}_{3}\right), 43.7\left(\mathrm{CH}_{2}\right), 73.5(\mathrm{CH}), 107.5(\mathrm{CH}), 111.9(\mathrm{CH}), 121.7(\mathrm{C})$, $127.3(2 \times \mathrm{CH}), 131.7(2 \times \mathrm{CH}), 135.6(\mathrm{C}), 140.8(\mathrm{C}), 142.7(\mathrm{CH}), 144.0(\mathrm{C}), 154.3(\mathrm{CO}) .{\mathrm{C}-\mathrm{NO}_{2} \text { was }}$ not observed under these experimental conditions. Anal. Calcld for $\mathrm{C}_{16} \mathrm{H}_{13} \mathrm{BrN}_{2} \mathrm{O}_{5}$ (393.19) $\mathrm{C}, 48.88 ; \mathrm{H}$, 3.33; N; 7.12. Found: C, 48.91; H, 3.39; N, 7.19.

4-(1-Hydroxy-2-(3-methyl-6-nitro-2-oxo-2,3-dihydrobenzo[d]oxazol-5-yl)ethyl)benzonitrile

$(5 \mathrm{c})$ : Yellow solid; mp $213{ }^{\circ} \mathrm{C} ;{ }^{1} \mathrm{H}-\mathrm{NMR}\left(\mathrm{CDCl}_{3}\right): \delta 2.27(\mathrm{~d}, J=3.0 \mathrm{~Hz}, 1 \mathrm{H}, \mathrm{OH}), 3.05(\mathrm{dd}, J=13.5 \mathrm{~Hz}$, $J=9.4 \mathrm{~Hz}, 1 \mathrm{H}, \mathrm{CH}), 3.46\left(\mathrm{~s}, 3 \mathrm{H}, \mathrm{NCH}_{3}\right), 3.57$ (dd, $\left.J=13.5 \mathrm{~Hz}, J=2.6 \mathrm{~Hz}, 1 \mathrm{H}, \mathrm{CH}\right), 5,15$ (dd, $J=9.4 \mathrm{~Hz}$, $J=2.6 \mathrm{HZ}, 1 \mathrm{H}, \mathrm{CH}), 6.93(\mathrm{~s}, 1 \mathrm{H}, \mathrm{CH}), 7.61(\mathrm{~d}, J=8.3 \mathrm{~Hz}, 2 \mathrm{H}, 2 \times \mathrm{CH}), 7.70(\mathrm{~d}, J=8.3 \mathrm{~Hz}, 2 \mathrm{H}$, $2 \times \mathrm{CH}) ; 7.98(\mathrm{~s}, 1 \mathrm{H}, \mathrm{CH}) .{ }^{13} \mathrm{C}-\mathrm{NMR}\left(\mathrm{CDCl}_{3}\right): \delta 28.6\left(\mathrm{NCH}_{3}\right), 43.8\left(\mathrm{CH}_{2}\right), 73.3(\mathrm{CH}), 107.6(\mathrm{CH}), 111.7$ (C), $112.0(\mathrm{CH}), 118.7(\mathrm{C}), 126.3(2 \times \mathrm{CH}), 131.5(\mathrm{C}), 132.5(2 \times \mathrm{CH}), 135.7(\mathrm{C}), 140.9(\mathrm{C}), 149.0(\mathrm{C})$, 154.2 (CO). $\mathrm{C}-\mathrm{NO}_{2}$ was not observed under these experimental conditions. HRMS (EI): calcd for $\mathrm{C}_{17} \mathrm{H}_{13} \mathrm{~N}_{3} \mathrm{O}_{5}\left(\mathrm{M}^{+}\right)$357.1193, found 357.1194.

5-(2-Hydroxy-2-(2-nitrophenyl)ethyl)-3-methyl-6-nitrobenzo[d]oxazol-2(3H)-one (5d): Brown solid; mp $130{ }^{\circ} \mathrm{C}$; ${ }^{1} \mathrm{H}-\mathrm{NMR}\left(\mathrm{CDCl}_{3}\right): \delta 3.36(\mathrm{dd}, J=13.8 \mathrm{~Hz}, J=8.8 \mathrm{~Hz}, 1 \mathrm{H}, \mathrm{CH}), 3.41\left(\mathrm{~s}, 3 \mathrm{H}, \mathrm{NCH}_{3}\right), 3.56(\mathrm{dd}$, 
$J=13.8 \mathrm{~Hz}, J=3.2 \mathrm{~Hz}, 1 \mathrm{H}, \mathrm{CH}), 5.47$ (dd, $J=8.8 \mathrm{~Hz}, J=3.2 \mathrm{~Hz}, 1 \mathrm{H}, \mathrm{CH}), 7.05$ (s, 1H, CH), 7.44 (t, $J=7.0 \mathrm{~Hz}, 1 \mathrm{H}, \mathrm{CH}), 7.65$ (t, $J=7.6 \mathrm{~Hz}, 1 \mathrm{H}, \mathrm{CH}), 7.73(\mathrm{~s}, 1 \mathrm{H}, \mathrm{CH}), 7.80$ (d, $J=7.0 \mathrm{~Hz}, 1 \mathrm{H}, \mathrm{CH}), 7.89$ (d, $J=7.6 \mathrm{~Hz}, 1 \mathrm{H}, \mathrm{CH}) .{ }^{13} \mathrm{C}-\mathrm{NMR}\left(\mathrm{CDCl}_{3}\right): \delta 28.5\left(\mathrm{NCH}_{3}\right), 40.6\left(\mathrm{CH}_{2}\right), 70.4(\mathrm{CH}), 107.1(\mathrm{CH}), 111.0(\mathrm{CH})$, $124.5(\mathrm{CH}), 128.4(\mathrm{CH}), 128.6(\mathrm{CH}), 131.1(\mathrm{C}), 133.9(\mathrm{CH}), 135.5(\mathrm{C}), 139.1(\mathrm{C}), 140.5(\mathrm{C}), 144.8(\mathrm{C})$, 147.4 (C), 154.3 (CO). HRMS (EI): calcd for $\mathrm{C}_{16} \mathrm{H}_{13} \mathrm{~N}_{3} \mathrm{O}_{7}\left(\mathrm{M}^{+}\right)$337.1092, found 337.1092.

5-(2-(2-Bromophenyl)-2-hydroxyethyl)-3-methyl-6-nitrobenzo[d]oxazol-2(3H)-one (5e): Yellow solid; mp $159{ }^{\circ} \mathrm{C}$; ${ }^{1} \mathrm{H}-\mathrm{NMR}$ (DMSO-d6): $\delta 3.29$ (s, 3H, NCH3), 3.30-3.33 (m, 2H, CH2), 5.51 (bs, 1H, CH), 7.15 (s, 1H, CH), $7.21(\mathrm{~d}, J=7.3 \mathrm{~Hz}, 1 \mathrm{H}, \mathrm{CH}), 7.40$ (t, $J=7.7 \mathrm{~Hz}, 1 \mathrm{H}, \mathrm{CH}), 7.51-7.54(\mathrm{~m}, 2 \mathrm{H}$, $2 \times \mathrm{CH}), 7.95(\mathrm{~s}, 1 \mathrm{H}, \mathrm{CH}) .{ }^{13} \mathrm{C}-\mathrm{NMR}\left(\mathrm{DMSO}-d_{6}\right): \delta 28.6\left(\mathrm{NCH}_{3}\right), 40.5\left(\mathrm{CH}_{2}\right), 71.35(\mathrm{CH}), 106.6(\mathrm{CH})$, $111.9(\mathrm{CH}), 121.4(\mathrm{C}), 128.1(\mathrm{CH}), 128.3(\mathrm{CH}), 129.3(\mathrm{C}), 130.5(\mathrm{CH}), 132.3(\mathrm{CH}), 135.4(\mathrm{C}), 140.1(\mathrm{C})$, 143.8 (C), 144.7 (C), 154.3 (CO). HRMS (EI): calcd for $\mathrm{C}_{16} \mathrm{H}_{13} \mathrm{BrN}_{2} \mathrm{O}_{5}\left(\mathrm{M}^{+}\right) 410.0346$, found 410.0347.

5-(2-(3-Bromophenyl)-2-hydroxyethyl)-3-methyl-6-nitrobenzo[d]oxazol-2(3H)-one (5f): Yellow solid; mp $154{ }^{\circ} \mathrm{C} ;{ }^{1} \mathrm{H}-\mathrm{NMR}\left(\mathrm{CDCl}_{3}\right): \delta 2.15(\mathrm{~d}, J=2.9 \mathrm{~Hz}, 1 \mathrm{H}, \mathrm{OH}), 3.13(\mathrm{dd}, J=13.6 \mathrm{~Hz}, J=9.0 \mathrm{~Hz}, 1 \mathrm{H}$, $\mathrm{CH}), 3.44\left(\mathrm{~s}, 3 \mathrm{H}, \mathrm{NCH}_{3}\right), 3.53(\mathrm{dd}, J=13.6 \mathrm{~Hz}, J=3.4 \mathrm{~Hz}, 1 \mathrm{H}, \mathrm{CH}), 5.08(\mathrm{dd}, J=9.0 \mathrm{~Hz}, J=3.4 \mathrm{~Hz}$, 1H, CH), 6.87 (s, 1H, CH), $7.29(\mathrm{~s}, 1 \mathrm{H}, \mathrm{CH}), 7.35-7.48(\mathrm{~m}, 2 \mathrm{H}, 2 \times \mathrm{CH}), 7.61-7.63(\mathrm{~m}, 1 \mathrm{H}, \mathrm{CH}), 7.95$ (s,1H, CH). ${ }^{13} \mathrm{C}-\mathrm{NMR}\left(\mathrm{CDCl}_{3}\right): \delta 28.5\left(\mathrm{NCH}_{3}\right), 43.7\left(\mathrm{CH}_{2}\right), 73.4(\mathrm{CH}), 107.5(\mathrm{CH}), 111.9(\mathrm{CH}), 122.8$ (C), $124.3(\mathrm{CH}), 128.7(\mathrm{CH}), 130.2(\mathrm{CH}), 131.0(\mathrm{CH}), 131.7(\mathrm{C}), 135.6(\mathrm{C}), 140.8(\mathrm{C}), 144.0(\mathrm{C}), 146.1$ (C), 154.3 (CO). Anal. Calcld for $\mathrm{C}_{16} \mathrm{H}_{13} \mathrm{BrN}_{2} \mathrm{O}_{5}$ (393.19) C, 48.88; H, 3.33; N, 7.12. Found: C, 49.11; H, 3.46; N, 7.28.

Ethyl 2-hydroxy-3-(3-methyl-6-nitro-2-oxo-2,3-dihydrobenzo[d]oxazol-5-yl)propanoate (5g): Yellow solid; mp $136{ }^{\circ} \mathrm{C} ;{ }^{1} \mathrm{H}-\mathrm{NMR}\left(\mathrm{CDCl}_{3}\right): \delta 1.32\left(\mathrm{t}, J=7.1 \mathrm{~Hz}, 3 \mathrm{H}, \mathrm{CH}_{3}\right), 2.99(\mathrm{~d}, J=5.3 \mathrm{~Hz}, 1 \mathrm{H}, \mathrm{OH}), 3.17(\mathrm{dd}$, $J=13.9 \mathrm{~Hz}, J=8.8 \mathrm{~Hz}, 1 \mathrm{H}, \mathrm{CH}), 3.46\left(\mathrm{~s}, 3 \mathrm{H}, \mathrm{NCH}_{3}\right), 3.68(\mathrm{dd}, J=13.9 \mathrm{~Hz}, J=3.7 \mathrm{~Hz}, 1 \mathrm{H}, \mathrm{CH}), 4.28$ (q, $\left.J=7.1 \mathrm{~Hz} 2 \mathrm{H}, \mathrm{CH}_{2}\right), 4.50-4.55(\mathrm{~m}, 1 \mathrm{H}, \mathrm{CH}), 7.02(\mathrm{~s}, 1 \mathrm{H}, \mathrm{CH}), 7.91(\mathrm{~s}, 1 \mathrm{H}, \mathrm{CH}) .{ }^{13} \mathrm{C}-\mathrm{NMR}\left(\mathrm{CDCl}_{3}\right)$ : $\delta 14.1\left(\mathrm{CH}_{3}\right), 28.6\left(\mathrm{NCH}_{3}\right), 37.9\left(\mathrm{CH}_{2}\right), 62.4\left(\mathrm{CH}_{2}\right), 70.1(\mathrm{CH}), 107.4(\mathrm{CH}), 111.6(\mathrm{CH}), 130.3(\mathrm{CH})$, $135.5(\mathrm{CH}), 140.8(\mathrm{CH}), 144.2(\mathrm{CH}), 154.2(\mathrm{CO}), 173.9(\mathrm{CO})$. Anal. Calcld for $\mathrm{C}_{13} \mathrm{H}_{14} \mathrm{~N}_{2} \mathrm{O}_{7}(310.26) \mathrm{C}$, 50.33 ; H, 4.55; N, 9.03. Found: C, 50.28; H, 4.54; N, 8.91

Diethyl 2-hydroxy-2-((3-methyl-6-nitro-2-oxo-2,3-dihydrobenzo[d]oxazol-5-yl)methyl)malonate (5h): Yellow solid; mp $111{ }^{\circ} \mathrm{C} ;{ }^{1} \mathrm{H}-\mathrm{NMR}\left(\mathrm{CDCl}_{3}\right): \delta 1.27\left(\mathrm{t}, J=7.1 \mathrm{~Hz}, 6 \mathrm{H}, 2 \mathrm{xCH}_{3}\right), 3.43\left(\mathrm{~s}, 3 \mathrm{H}, \mathrm{NCH}_{3}\right)$, 3.85 (bs, 1H, OH), 3.88 (s, 2H, CH 2$), 4.11-4.34$ (m, 4H, $\left.\mathrm{CH}_{2}\right), 7.14(\mathrm{~s}, 1 \mathrm{H}, \mathrm{CH}), 7.75(\mathrm{~s}, 1 \mathrm{H}, \mathrm{CH})$. ${ }^{13} \mathrm{C}-\mathrm{NMR}\left(\mathrm{CDCl}_{3}\right): \delta 13.9\left(2 \times \mathrm{CH}_{3}\right), 28.5\left(\mathrm{NCH}_{3}\right), 35.5\left(2 \times \mathrm{CH}_{2}\right), 63.1\left(\mathrm{CH}_{2}\right), 78.4(\mathrm{C}-\mathrm{OH}), 107.2$ $(\mathrm{CH}), 111.8(\mathrm{CH}), 127.1(\mathrm{C}), 134.8(\mathrm{C}), 140.8(\mathrm{C}), 145.6(\mathrm{C}), 154.2(\mathrm{CO}), 169.4(2 \times \mathrm{CO})$. Anal. Calcld for $\mathrm{C}_{16} \mathrm{H}_{18} \mathrm{~N}_{2} \mathrm{O} 9$ (382.32) C, 50.26, H, 4.75, N, 7.33. Found: C, 50.25, H, 4.83, N, 7.18.

5-((1-Hydroxy-2-oxo-1,2-dihydroacenaphthylen-1-yl)methyl)-3-methyl-6-nitrobenzo[d]oxazol-2(3H)-one (5i): Green solid; mp $204{ }^{\circ} \mathrm{C} ;{ }^{1} \mathrm{H}-\mathrm{NMR}\left(\mathrm{CDCl}_{3}\right): \delta 3.45\left(\mathrm{~s}, 3 \mathrm{H}, \mathrm{NCH}_{3}\right), 3.64(\mathrm{~d}, J=14.0 \mathrm{~Hz}, 1 \mathrm{H}, \mathrm{CH})$, $3.84(\mathrm{~d}, J=14.0 \mathrm{~Hz}, 1 \mathrm{H}, \mathrm{CH}), 7.12(\mathrm{~s}, 1 \mathrm{H}, \mathrm{CH}), 7.27$ (d, $J=7.3 \mathrm{~Hz}, 1 \mathrm{H}, \mathrm{CH}), 7.61$ (dd, $J=8.0 \mathrm{~Hz}$, $J=7.3 \mathrm{~Hz}, 1 \mathrm{H}, \mathrm{CH}), 7.77(\mathrm{dd}, J=7.8 \mathrm{~Hz}, J=7.3 \mathrm{~Hz}, 1 \mathrm{H}, \mathrm{CH}), 7.88(\mathrm{~s}, 1 \mathrm{H}, \mathrm{CH}), 7.89-7.98(\mathrm{~m}, 2 \mathrm{H}$, $2 \times \mathrm{CH}), 8.15(\mathrm{~d}, J=8.0 \mathrm{~Hz}, 1 \mathrm{H}, \mathrm{CH}) .{ }^{13} \mathrm{C}-\mathrm{NMR}\left(\mathrm{CDCl}_{3}\right): \delta 28.6\left(\mathrm{NCH}_{3}\right), 40.9\left(\mathrm{CH}_{2}\right), 79.8(\mathrm{C}-\mathrm{OH})$, $107.5(\mathrm{CH}), 112.4(\mathrm{CH}), 120.3(\mathrm{CH}), 122.7(\mathrm{CH}), 125.9(\mathrm{CH}), 128.6(\mathrm{CH}), 128.7(\mathrm{CH}+\mathrm{C}), 130.1(\mathrm{C})$, 
130.7 (C), 132.4 (CH), 135.3 (C), 138.7 (C), 140.7 (C), 141.0 (C), 144.5 (C), 154.3 (CO); 203.8 (CO). Anal. Calcld for $\mathrm{C}_{21} \mathrm{H}_{14} \mathrm{~N}_{2} \mathrm{O}_{6}(390.35)$ C, 64.62, H, 3.62, N, 7.18. Found: C, 64.15, H, 3.72, N, 7.05.

5-((3-Hydroxy-1-methyl-2-oxoindolin-3-yl)methyl)-3-methyl-6-nitrobenzo[d]oxazol-2(3H)-one

Yellow solid; mp $253{ }^{\circ} \mathrm{C} ;{ }^{1} \mathrm{H}-\mathrm{NMR}$ (DMSO-d6): $\delta 3.03\left(\mathrm{~s}, 3 \mathrm{H}, \mathrm{NCH}_{3}\right), 3.31\left(\mathrm{~s}, 3 \mathrm{H}, \mathrm{NCH}_{3}\right), 3.36(\mathrm{~d}$, $J=13.7 \mathrm{~Hz}, 1 \mathrm{H}, \mathrm{CH}), 3.66$ (d, $J=13.7 \mathrm{~Hz}, 1 \mathrm{H}, \mathrm{CH}), 6.19$ (s, 1H, CH), $6.78(\mathrm{~d}, J=6.8 \mathrm{~Hz}, 1 \mathrm{H}, \mathrm{CH})$, 6.91-6.95 (m, 2H, $2 \times \mathrm{CH}), 7.13(\mathrm{~s}, 1 \mathrm{H}, \mathrm{CH}), 7.24-7.31(\mathrm{~m}, 1 \mathrm{H}, \mathrm{CH}) .{ }^{13} \mathrm{C}-\mathrm{NMR}$ (DMSO-d6): $\delta 26.0$ $\left(\mathrm{NCH}_{3}\right) ; 28.5\left(\mathrm{NCH}_{3}\right), 75.4(\mathrm{C}-\mathrm{OH}), 106.7(\mathrm{CH}), 108.6(\mathrm{CH}), 112.9(\mathrm{CH}), 122.3(\mathrm{CH}), 123.9(\mathrm{CH})$, 127.6 (C), 129.4 (CH), 130.5 (C), 135.0 (C), 140.3 (C), 142.8 (C), 144.7 (C), 154.3 (CO), 176.8 (CO). $\mathrm{C}-\mathrm{NO}_{2}$ was not observed under these experimental conditions. Anal. Calcld for $\mathrm{C}_{18} \mathrm{H}_{15} \mathrm{~N}_{3} \mathrm{O}_{6}(369.33) \mathrm{C}$, 58.54, H, 4.09, N, 11.38. Found: C, 58.26, H, 4.25, N, 11.01.

1-(3-Methyl-6-nitro-2-oxo-2,3-dihydrobenzo[d]oxazol-5-yl)-2-(4-nitrophenyl)ethyl 4-nitrobenzoate (6): Yellow solid; mp $305{ }^{\circ} \mathrm{C}$; ${ }^{1} \mathrm{H}-\mathrm{NMR}\left(\mathrm{CDCl}_{3}\right)$ : $\delta 3.35$ (s, $\left.3 \mathrm{H}, \mathrm{NCH}_{3}\right), 3.73\left(\mathrm{~d}, J=6.2 \mathrm{~Hz}, 2 \mathrm{H}, \mathrm{CH}_{2}\right), 6.44$ $(\mathrm{t}, J=6.2 \mathrm{~Hz}, 1 \mathrm{H}, \mathrm{CH}), 6.84(\mathrm{~s}, 1 \mathrm{H}, \mathrm{CH}), 7.68(\mathrm{~d}, J=8.5 \mathrm{~Hz}, 2 \mathrm{H}, 2 \times \mathrm{CH}), 7.97(\mathrm{~s}, 1 \mathrm{H}, \mathrm{CH}), 8.17(\mathrm{~d}$, $J=8.8 \mathrm{~Hz}, 2 \mathrm{H}, 2 \times \mathrm{CH}), 8.28(\mathrm{~d}, J=8.5 \mathrm{~Hz}, 1 \mathrm{H}, 2 \times \mathrm{CH}), 8.32(\mathrm{~d}, J=8.5 \mathrm{~Hz}, 1 \mathrm{H}, 2 \times \mathrm{CH}) .{ }^{13} \mathrm{C}-\mathrm{NMR}$ $\left(\mathrm{CDCl}_{3}\right): \delta 28.5\left(\mathrm{NCH}_{3}\right), 40.8\left(\mathrm{CH}_{2}\right), 76.4(\mathrm{CH}), 108.1(\mathrm{CH}), 110.7(\mathrm{CH}), 114.1(\mathrm{C}), 123.8(2 \times \mathrm{CH})$, $124.3(2 \times \mathrm{CH}), 127.1(2 \times \mathrm{CH}), 129.6(\mathrm{C}), 130.7(2 \times \mathrm{CH}), 134.4(\mathrm{C}), 135.9(\mathrm{C}), 141.2(\mathrm{C}), 145.9(\mathrm{C})$, 148.1 (C), 150.9 (C), 153.8 (CO). HRMS (EI): calcd for $\mathrm{C}_{23} \mathrm{H}_{16} \mathrm{~N}_{4} \mathrm{O}_{10}\left(\mathrm{M}^{+}\right)$526.1205, found 526.1209.

\subsection{General Procedure for the Reaction of $\mathbf{3}$ and Aromatic Carbonyl Derivatives 4a-f, $\alpha$-Carbonyl} Ester 4g, Ketomalonate 4h, Acenaphtenedione 4i and Keto-lactam 4j Using TDAE

A solution of $3(0.5 \mathrm{~g}, 1.36 \mathrm{mmol})$ in anhydrous DMF $(10 \mathrm{~mL})$ and the corresponding carbonyl derivative $4 \mathbf{a}-\mathbf{j}$ (4.098 mmol, 3 equivalents) were placed under nitrogen at $-20{ }^{\circ} \mathrm{C}$ in a two-necked flask equipped with a silica-gel drying tube and a nitrogen inlet. The solution was stirred and maintained at this temperature for $30 \mathrm{~min}$ and then the TDAE $(0.48 \mathrm{~mL}, 2.049 \mathrm{mmol}, 1.5$ equivalent $)$ was added dropwise via a syringe. A red color immediately developed with the formation of a fine white precipitate. The solution was vigorously stirred at $-20^{\circ} \mathrm{C}$ for $1 \mathrm{~h}$ and then warmed to $\mathrm{rt}$ for $2 \mathrm{~h}$. After this time TLC analysis (dichloromethane) clearly showed that 3 was totally consumed. The orange-red turbid solution was filtered (to remove the octamethyloxamidinium dibromide) and hydrolyzed with $80 \mathrm{~mL}$ of $\mathrm{H}_{2} \mathrm{O}$. The aqueous solution was extracted with toluene $(3 \times 40 \mathrm{~mL})$, the combined organic layers washed with $\mathrm{H}_{2} \mathrm{O}(3 \times 40 \mathrm{~mL})$ and dried over $\mathrm{MgSO}_{4}$. Evaporation of the solvent left an orange viscous liquid as crude product. Purification by silica gel chromatography and recrystallization in ethyl alcohol solvent gave the corresponding oxiranes $7 \mathbf{a}-\mathbf{j}$.

3-Methyl-6-nitro-5-(3-(4-nitrophenyl)oxiran-2-yl)benzo[d]oxazol-2(3H)-one (7a) trans-isomer: Yellow solid; mp $224{ }^{\circ} \mathrm{C} ;{ }^{1} \mathrm{H}-\mathrm{NMR}\left(\mathrm{CDCl}_{3}\right)$ : $\delta 3.52\left(\mathrm{~s}, 3 \mathrm{H}, \mathrm{NCH}_{3}\right), 3.92(\mathrm{~d}, J=1.9 \mathrm{~Hz}, 1 \mathrm{H}, \mathrm{CH}), 4.54(\mathrm{~d}$, $J=1.9 \mathrm{~Hz}, 1 \mathrm{H}, \mathrm{CH}), 7.33(\mathrm{~s}, \mathrm{H}, \mathrm{CH}), 7.60(\mathrm{~d}, J=8.7 \mathrm{~Hz}, 2 \mathrm{H}, 2 \times \mathrm{CH}), 8.13(\mathrm{~s}, 1 \mathrm{H}, \mathrm{CH}), 8.30(\mathrm{~d}$, $J=8.7 \mathrm{~Hz}, 2 \mathrm{H}, 2 \times \mathrm{CH}) .{ }^{13} \mathrm{C}-\mathrm{NMR}\left(\mathrm{CDCl}_{3}\right): \delta 28.8\left(\mathrm{NCH}_{3}\right), 61.0(\mathrm{CH}), 61.1(\mathrm{CH}), 105.7(\mathrm{CH}), 107.4$ $(\mathrm{CH}), 124.0(2 \times \mathrm{CH}), 126.6(2 \times \mathrm{CH}), 131.8(\mathrm{C}), 137.28(\mathrm{C}), 141.6(\mathrm{C}), 142.1(\mathrm{C}), 143.0(\mathrm{C}), 148.2$ (CO). C-NO $\mathrm{N}_{2}$ was not observed under these experimental conditions. HRMS (EI): calcd for $\mathrm{C}_{16} \mathrm{H}_{11} \mathrm{~N}_{3} \mathrm{O}_{7}$ $\left(\mathrm{M}^{+}\right)$375.0935, found 375.0943. 
5-(3-(4-Bromophenyl)oxiran-2-yl)-3-methyl-6-nitrobenzo[d]oxazol-2(3H)-one (7b) trans-isomer: Yellow solid; mp $209{ }^{\circ} \mathrm{C}$; ${ }^{1} \mathrm{H}-\mathrm{NMR}\left(\mathrm{CDCl}_{3}\right): \delta 3.51$ (s, 3H, $\left.\mathrm{NCH}_{3}\right), 3.77(\mathrm{~d}, J=1.9 \mathrm{~Hz}, 1 \mathrm{H}, \mathrm{CH}), 3.54$ $(\mathrm{d}, J=1.9 \mathrm{~Hz}, 1 \mathrm{H}, \mathrm{CH}), 7.28(\mathrm{~d}, J=8.4 \mathrm{~Hz}, 2 \mathrm{H}, 2 \times \mathrm{CH}), 7.31(\mathrm{~s}, 1 \mathrm{H}, \mathrm{CH}), 7.54(\mathrm{~d}, J=8.4 \mathrm{~Hz}, 2 \mathrm{H}, 2 \times \mathrm{CH})$, $8.11(\mathrm{~s}, 1 \mathrm{H}, \mathrm{CH}) .{ }^{13} \mathrm{C}-\mathrm{NMR}\left(\mathrm{CDCl}_{3}\right): \delta 28.7\left(\mathrm{NCH}_{3}\right), 60.5(\mathrm{CH}), 61.7(\mathrm{CH}), 105.6(\mathrm{CH}), 107.3(\mathrm{CH})$, $122.8(\mathrm{C}), 127.5(2 \times \mathrm{CH}), 131.9(2 \times \mathrm{CH}), 132.5(\mathrm{C}), 134.8(\mathrm{C}), 137.0(\mathrm{C}), 141.3(\mathrm{C}), 154.1(\mathrm{CO})$. Anal. Calcld for $\mathrm{C}_{16} \mathrm{H}_{11} \mathrm{BrN}_{2} \mathrm{O}_{5}(391.17)$ C, 49.13; H, 2.83; N, 7.16. Found: C, 49.27; H, 2.92; N, 7.85.

4-(3-(3-Methyl-6-nitro-2-oxo-2,3-dihydrobenzo[d]oxazol-5-yl)oxiran-2-yl)benzonitrile (7c) trans-isomer: Yellow solid; $\mathrm{mp} 213{ }^{\circ} \mathrm{C}$; ${ }^{1} \mathrm{H}-\mathrm{NMR}\left(\mathrm{CDCl}_{3}\right): \delta 3.51\left(\mathrm{~s}, 3 \mathrm{H}, \mathrm{NCH}_{3}\right), 3.86(\mathrm{~d}, J=1.8 \mathrm{~Hz}, \mathrm{H}, \mathrm{CH}), 4.52(\mathrm{~d}$, $J=1.8 \mathrm{~Hz}, \mathrm{H}, \mathrm{CH}), 7.32(\mathrm{~s}, 1 \mathrm{H}, \mathrm{CH}), 7.53(\mathrm{~d}, J=8.3 \mathrm{~Hz}, 2 \mathrm{H}, 2 \times \mathrm{CH}), 7.72(\mathrm{~d}, J=8.3 \mathrm{~Hz}, 2 \mathrm{H}, 2 \times \mathrm{CH})$, $8.12(\mathrm{~s}, 1 \mathrm{H}, \mathrm{CH}) .{ }^{13} \mathrm{C}-\mathrm{NMR}\left(\mathrm{CDCl}_{3}\right): \delta 28.7\left(\mathrm{NCH}_{3}\right), 61.0(\mathrm{CH}), 61.2(\mathrm{CH}), 105.7(\mathrm{CH}), 107.3(\mathrm{CH})$, $112.6(\mathrm{C}), 118.5(\mathrm{C}), 126.5(2 \times \mathrm{CH}), 131.9(\mathrm{C}), 132.5(2 \times \mathrm{CH}), 137.1(\mathrm{C}), 141.1(\mathrm{C}), 141.5(\mathrm{C})$, 142.1(C); 154.1 (CO). HRMS (EI): calcd for $\mathrm{C}_{17} \mathrm{H}_{11} \mathrm{~N}_{3} \mathrm{O}_{5}\left(\mathrm{M}^{+}\right)$355.1037, found 355.1036.

3-Methyl-6-nitro-5-(3-(2-nitrophenyl)oxiran-2-yl)benzo[d]oxazol-2(3H)-one (7d) trans-isomer: yellow solid; mp $215{ }^{\circ} \mathrm{C} ;{ }^{1} \mathrm{H}-\mathrm{NMR}\left(\mathrm{CDCl}_{3}\right)$ : $\delta 3.52\left(\mathrm{~s}, 3 \mathrm{H}, \mathrm{NCH}_{3}\right), 4.54(\mathrm{~d}, J=2.0 \mathrm{~Hz}, 1 \mathrm{H}, \mathrm{CH}), 4.60(\mathrm{~d}$, $J=2.0 \mathrm{~Hz}, 1 \mathrm{H}, \mathrm{CH}), 7.36(\mathrm{~s}, 1 \mathrm{H}, \mathrm{CH}), 7.52-7.61(\mathrm{~m}, 1 \mathrm{H}, \mathrm{CH}), 7.75-7.77(\mathrm{~m}, 2 \mathrm{H}, 2 \times \mathrm{CH}), 8.14(\mathrm{~s}, 1 \mathrm{H}$, $\mathrm{CH}), 8.23(\mathrm{~d}, J=8.0 \mathrm{~Hz}, 1 \mathrm{H}, \mathrm{CH}) .{ }^{13} \mathrm{C}-\mathrm{NMR}\left(\mathrm{CDCl}_{3}\right): \delta 28.7\left(\mathrm{NCH}_{3}\right), 59.9(\mathrm{CH}), 60.0(\mathrm{CH}), 105.5(\mathrm{CH})$, 107.6 (CH), $125.2(\mathrm{CH}), 126.9(\mathrm{CH}), 129.2(\mathrm{C}), 131.8(\mathrm{CH}), 132.5(\mathrm{C}), 134.4(\mathrm{CH}), 137.0(\mathrm{C}), 141.5(\mathrm{C})$, 142.6 (C), 147.9 (C), 154.2 (CO). HRMS (EI): calcd for $\mathrm{C}_{16} \mathrm{H}_{11} \mathrm{~N}_{3} \mathrm{O}_{7}\left(\mathrm{M}^{+}\right)$375.0935, found 375.0940.

3-Methyl-6-nitro-5-(3-(2-nitrophenyl)oxiran-2-yl)benzo[d]oxazol-2(3H)-one (7d) cis-isomer: Beige solid; mp $166{ }^{\circ} \mathrm{C} ;{ }^{1} \mathrm{H}-\mathrm{NMR}\left(\mathrm{CDCl}_{3}\right): \delta 3.38\left(\mathrm{~s}, 3 \mathrm{H}, \mathrm{NCH}_{3}\right), 5.14(\mathrm{~d}, J=4.9 \mathrm{~Hz}, 1 \mathrm{H}, \mathrm{CH}), 5.17(\mathrm{~d}, J=4.9 \mathrm{~Hz}$, $1 \mathrm{H}, \mathrm{CH}), 7.03(\mathrm{~s}, 1 \mathrm{H}, \mathrm{CH}), 7.30-7.40(\mathrm{~m}, 1 \mathrm{H}, \mathrm{CH}), 7.44-7.46(\mathrm{~m}, 2 \mathrm{H}, 2 \times \mathrm{CH}), 7.86-7.90(\mathrm{~m}, 2 \mathrm{H}$, $2 \times \mathrm{CH}) .{ }^{13} \mathrm{C}-\mathrm{NMR}\left(\mathrm{CDCl}_{3}\right): \delta 28.6\left(\mathrm{NCH}_{3}\right), 59.0(2 \times \mathrm{CH}), 107.3(\mathrm{CH}), 107.4(\mathrm{CH}), 124.7(\mathrm{CH}), 128.8$ (CH), $128.9(\mathrm{C}), 129.2(\mathrm{CH}), 129.4(\mathrm{C}), 132.7(\mathrm{CH}), 135.9(\mathrm{C}), 141.2(\mathrm{C}), 148.5(\mathrm{C}), 153.9(\mathrm{CO})$. Anal. Calcld for $\mathrm{C}_{16} \mathrm{H}_{11} \mathrm{~N}_{3} \mathrm{O}_{7}(357.27)$ C, 53.79; H, 3.10; N, 11.76. Found: C, 53.48; H, 3.30; N, 11.44 .

5-(3-(2-Bromophenyl)oxiran-2-yl)-3-methyl-6-nitrobenzo[d]oxazol-2(3H)-one (7e) trans-isomer: Green solid; mp $203{ }^{\circ} \mathrm{C} ;{ }^{1} \mathrm{H}-\mathrm{NMR}\left(\mathrm{CDCl}_{3}\right)$ : $\delta 3.52$ (s, 3H, NCH$), 4.09$ (d, $\left.J=1.9 \mathrm{~Hz}, 1 \mathrm{H}, \mathrm{CH}\right), 4.57$ (d, $J=1.9 \mathrm{~Hz}, 1 \mathrm{H}, \mathrm{CH}), 7.20-7.29(\mathrm{~m}, 1 \mathrm{H}, \mathrm{CH}), 7.36(\mathrm{~s}, 1 \mathrm{H}, \mathrm{CH}), 7.38-7.47(\mathrm{~m}, 2 \mathrm{H}, 2 \times \mathrm{CH}), 7.60(\mathrm{~d}$, $J=7.5 \mathrm{~Hz}, 1 \mathrm{H}, \mathrm{CH}), 8.12(\mathrm{~s}, 1 \mathrm{H}, \mathrm{CH}) .{ }^{13} \mathrm{C}-\mathrm{NMR}\left(\mathrm{CDCl}_{3}\right): \delta 28.7\left(\mathrm{NCH}_{3}\right), 60.1(\mathrm{CH}), 62.0(\mathrm{CH}), 105.6$ $(\mathrm{CH}), 107.4(\mathrm{CH}), 123.1(\mathrm{C}), 126.1(\mathrm{CH}), 127.8(\mathrm{CH}), 130.0(\mathrm{CH}), 132.2(\mathrm{C}), 132.7(\mathrm{CH}), 135.2(\mathrm{C}), 137.0$ (C), 141.4 (C), 142.4 (C), 154.2 (CO). Anal. Calcld for $\mathrm{C}_{16} \mathrm{H}_{11} \mathrm{BrN}_{2} \mathrm{O}_{5}$ (391.17) C, 49.13; H, 2.83; N, 7.16. Found: C, 49.27; H, 2.93; N, 7.17.

5-(3-(2-Bromophenyl)oxiran-2-yl)-3-methyl-6-nitrobenzo [d] oxazol-2(3H)-one (7e) cis-isomer: Green solid; mp $151{ }^{\circ} \mathrm{C}$; ${ }^{1} \mathrm{H}-\mathrm{NMR}\left(\mathrm{CDCl}_{3}\right): \delta 3.42\left(\mathrm{~s}, 3 \mathrm{H}, \mathrm{NCH}_{3}\right), 4.71(\mathrm{~d}, J=4.4 \mathrm{~Hz}, 1 \mathrm{H}, \mathrm{CH}), 5.17(\mathrm{~d}$, $J=4.4 \mathrm{~Hz}, 1 \mathrm{H}, \mathrm{CH}), 6.98-7.14(\mathrm{~m}, 3 \mathrm{H}, 3 \times \mathrm{CH}), 7.16(\mathrm{~s}, 1 \mathrm{H}, \mathrm{CH}), 7.36-7.42(\mathrm{~m}, 1 \mathrm{H}, \mathrm{CH}), 7.93(\mathrm{~s}, 1 \mathrm{H}$, $\mathrm{CH}) .{ }^{13} \mathrm{C}-\mathrm{NMR}\left(\mathrm{CDCl}_{3}\right): \delta 28.6\left(\mathrm{NCH}_{3}\right), 59.3(\mathrm{CH}), 61.0(\mathrm{CH}), 107.3(\mathrm{CH}), 107.9(\mathrm{CH}), 122.5(\mathrm{C}), 126.4$ $(\mathrm{CH}), 128.0(\mathrm{CH}), 129.4(\mathrm{C}), 129.6(\mathrm{CH}), 132.7(\mathrm{CH}), 132.9(\mathrm{C}), 135.8(\mathrm{C}), 141.1(\mathrm{C}), 142.5(\mathrm{C}), 154.0$ (CO). Anal. Calcld for $\mathrm{C}_{16} \mathrm{H}_{11} \mathrm{BrN}_{2} \mathrm{O}_{5}(391.17) \mathrm{C}, 49.13 ; \mathrm{H}, 2.83 ; \mathrm{N}, 7.16$. Found: C, 49.42; H, 3.02; N, 7.28. 
5-(3-(3-Bromophenyl)oxiran-2-yl)-3-methyl-6-nitrobenzo[d]oxazol-2(3H)-one (7f) trans-isomer: Beige solid; mp $165{ }^{\circ} \mathrm{C} ;{ }^{1} \mathrm{H}-\mathrm{NMR}\left(\mathrm{CDCl}_{3}\right): \delta 3.51(\mathrm{~s}, 3 \mathrm{H}, 3 \mathrm{H}, \mathrm{NCH}), 3.77(\mathrm{~d}, J=1.9 \mathrm{~Hz}, 1 \mathrm{H}, \mathrm{CH}), 4.55(\mathrm{~d}$, $J=1.9 \mathrm{~Hz}, 1 \mathrm{H}, \mathrm{CH}), 7.24-7.28(\mathrm{~m}, 1 \mathrm{H}, \mathrm{CH}), 7.32$ (s, 1H, CH), 7.33-7.38 (m, 1H, CH), 7.49-7.54 (m, $2 \mathrm{H}, 2 \times \mathrm{CH}), 8.11(\mathrm{~s}, 1 \mathrm{H}, \mathrm{CH}) .{ }^{13} \mathrm{C}-\mathrm{NMR}(\mathrm{CDCl} 3): \delta 28.7\left(\mathrm{NCH}_{3}\right), 60.6(\mathrm{CH}), 61.4(\mathrm{CH}), 105.7(\mathrm{CH})$, $107.3(\mathrm{CH}), 122.8(\mathrm{C}), 124.6(\mathrm{CH}), 128.7(\mathrm{CH}), 130.2(\mathrm{CH}), 131.9(\mathrm{CH}), 132.3(\mathrm{C}), 137.0(\mathrm{C}), 138.1$ (C), 141.4 (C), 142.1 (C), 154.1 (CO). Anal. Calcld for $\mathrm{C}_{16} \mathrm{H}_{11} \mathrm{BrN}_{2} \mathrm{O}_{5}$ (391.17) C, 49.13; H, 2.83; N, 7.16. Found: C, 49.30; H, 2.97; N, 7.10.

Ethyl 3-(3-methyl-6-nitro-2-oxo-2,3-dihydrobenzo[d]oxazol-5-yl)oxirane-2-carboxylate (7g) trans-isomer: Light yellow needles; mp $199{ }^{\circ} \mathrm{C} ;{ }^{1} \mathrm{H}-\mathrm{NMR}\left(\mathrm{CDCl}_{3}\right): \delta 1.36$ (t, $\left.J=7.2 \mathrm{~Hz}, \mathrm{H}, \mathrm{CH}\right) ; 3.38(\mathrm{~d}, J=1.9 \mathrm{~Hz}$, $3 \mathrm{H}, \mathrm{CH}) ; 3.48$ (s, 3H, NCH$) ; 4,35$ (q, $\left.J=7.2 \mathrm{~Hz}, 2 \mathrm{H}, \mathrm{CH}_{2}\right) ; 4,75$ (d, J=1.9 Hz, 1H, CH); 7.19 (s, 1H, $\mathrm{CH}) ; 8.12(\mathrm{~s}, 1 \mathrm{H}, \mathrm{CH}) .{ }^{13} \mathrm{C}-\mathrm{NMR}\left(\mathrm{CDCl}_{3}\right): \delta 14.1\left(\mathrm{CH}_{3}\right), 28.8\left(\mathrm{NCH}_{3}\right), 56.0(\mathrm{CH}), 56.6(\mathrm{CH}), 62.2$ $\left(\mathrm{CH}_{2}\right), 105.8(\mathrm{CH}), 107.4(\mathrm{CH}), 130.9(\mathrm{C}), 137.0(\mathrm{C}), 141.6(\mathrm{C}), 142.2(\mathrm{C}), 154.0(\mathrm{CO}), 167.2(\mathrm{CO})$. HRMS (EI): calcd for $\mathrm{C}_{13} \mathrm{H}_{12} \mathrm{~N}_{2} \mathrm{O}_{7}\left(\mathrm{M}^{+}\right)$309.0717, found 309.0713.

Diethyl 3-(3-methyl-6-nitro-2-oxo-2,3-dihydrobenzo[d]oxazol-5-yl)oxirane-2,2-dicarboxylate trans-isomer: Dark brown; mp $118{ }^{\circ} \mathrm{C} ;{ }^{1} \mathrm{H}-\mathrm{NMR}\left(\mathrm{CDCl}_{3}\right): \delta 0.98\left(\mathrm{t}, J=7.2 \mathrm{~Hz}, 3 \mathrm{H}, \mathrm{CH}_{3}\right), 1.37$ (t, $\left.J=7.2 \mathrm{~Hz}, 3 \mathrm{H}, \mathrm{CH}_{3}\right), 3.48\left(\mathrm{~s}, 3 \mathrm{H}, \mathrm{NCH}_{3}\right), 3.98\left(\mathrm{q}, J=7.2 \mathrm{~Hz}, 2 \mathrm{H}, \mathrm{CH}_{2}\right), 4.39\left(\mathrm{q}, J=7.2 \mathrm{~Hz}, 2 \mathrm{H}, \mathrm{CH}_{2}\right)$, $5.14(\mathrm{~s}, 1 \mathrm{H}, \mathrm{CH}), 7.24(\mathrm{~s}, 1 \mathrm{H}, \mathrm{CH}), 8.12(\mathrm{~s}, 1 \mathrm{H}, \mathrm{CH}) .{ }^{13} \mathrm{C}-\mathrm{NMR}\left(\mathrm{CDCl}_{3}\right): \delta 13.8\left(\mathrm{CH}_{3}\right), 14.0\left(\mathrm{CH}_{3}\right), 28.9$ $\left(\mathrm{NCH}_{3}\right), 61.2(\mathrm{CH}), 62.2\left(\mathrm{CH}_{2}\right), 63.3\left(\mathrm{CH}_{2}\right), 107.2(\mathrm{CH}), 107.4(\mathrm{CH}), 127.9(\mathrm{C}), 136.7(\mathrm{C}), 141.9(\mathrm{C}), 142.3$ (C), 153.9 (C), 163.3 (CO), 164.6 (CO). Anal. Calcld for $\mathrm{C}_{16} \mathrm{H}_{16} \mathrm{~N}_{2} \mathrm{O} 9$ (380.31) C, 50.53; H, 4.24; N, 7.37. Found: C, 50.96; H, 4.54; N, 7.25.

3-Methyl-6-nitro-5-(2-oxo-2H-spiro[acenaphthylene-1,2'-oxiran]-3'-yl)benzo[d]oxazol-2(3H)-one like-isomer: Yellow solid; mp $235{ }^{\circ} \mathrm{C} ;{ }^{1} \mathrm{H}-\mathrm{NMR}\left(\mathrm{CDCl}_{3}\right)$ : $\delta 3.59\left(\mathrm{~s}, 3 \mathrm{H}, \mathrm{NCH}_{3}\right), 5.29(\mathrm{~s}, 1 \mathrm{H}, \mathrm{CH}), 7.62$ $\left(\mathrm{d}, J=6.8 \mathrm{~Hz}, 1 \mathrm{H}, \mathrm{CH}_{2}\right), 7.74(\mathrm{~s}, 2 \mathrm{H}, 2 \times \mathrm{CH}) ; 7.77-7.80(\mathrm{~m}, 1 \mathrm{H}, \mathrm{CH}), 7.85\left(\mathrm{~d}, J=6.7 \mathrm{~Hz}, 1 \mathrm{H}, \mathrm{CH}_{2}\right)$, $8.01\left(\mathrm{~d}, J=8.4 \mathrm{~Hz}, 1 \mathrm{H}, \mathrm{CH}_{2}\right), 8.06(\mathrm{~s}, 1 \mathrm{H}, \mathrm{CH}), 8.19(\mathrm{~d}, J=8.1 \mathrm{~Hz}, 1 \mathrm{H}, \mathrm{CH}) .{ }^{13} \mathrm{C}-\mathrm{NMR}\left(\mathrm{CDCl}_{3}\right): \delta 28.9$ $\left(\mathrm{NCH}_{3}\right), 65.8(\mathrm{CH}), 67.1(\mathrm{C}), 106.8(\mathrm{CH}), 108.9(\mathrm{CH}), 118.9(\mathrm{CH}), 122.1(\mathrm{CH}), 126.5(\mathrm{CH}), 128.3(\mathrm{CH})$, $128.7(\mathrm{CH}), 129.2(\mathrm{C}), 130.4(\mathrm{C}), 131.2(\mathrm{C}), 132.1(\mathrm{C}), 132.3(\mathrm{CH}), 136.5(\mathrm{C}), 141.4(\mathrm{C}), 141.7(\mathrm{C})$, 142.5 (C), 154.2 (CO), 196.0 (CO). Anal. Calcld for $\mathrm{C}_{21} \mathrm{H}_{12} \mathrm{~N}_{2} \mathrm{O}_{6}$ (388.33) C, 64.95; H, 3.11; N, 7.21. Found: C, 64.08; H, 3.26; N, 6.85 .

3-Methyl-6-nitro-5-(2-oxo-2H-spiro[acenaphthylene-1,2'-oxiran]-3'-yl)benzo[d]oxazol-2(3H)-one unlike-isomer: Beige solid; mp $201{ }^{\circ} \mathrm{C} ;{ }^{1} \mathrm{H}-\mathrm{NMR}\left(\mathrm{CDCl}_{3}\right)$ : $\delta 3.63\left(\mathrm{~s}, 3 \mathrm{H}, \mathrm{NCH}_{3}\right), 5.30(\mathrm{~s}, 1 \mathrm{H}, \mathrm{CH}), 6.34$ $\left(\mathrm{d}, J=6.8 \mathrm{~Hz}, 1 \mathrm{H}, \mathrm{CH}_{2}\right), 7.32(\mathrm{~d}, J=6,7 \mathrm{~Hz}, 1 \mathrm{H}, \mathrm{CH}), 7.67(\mathrm{~s}, 1 \mathrm{H}, \mathrm{CH}), 7.77-7.88(\mathrm{~m}, 2 \mathrm{H}, 2 \times \mathrm{CH})$, $8.07(\mathrm{~s}, 1 \mathrm{H}, \mathrm{CH}), 8.12(\mathrm{~d}, J=1.7 \mathrm{~Hz}, 1 \mathrm{H}, \mathrm{CH}), \mathrm{CH}), 8.16(\mathrm{~d}, J=3.2 \mathrm{~Hz}, 1 \mathrm{H}, \mathrm{CH}) .{ }^{13} \mathrm{C}-\mathrm{NMR}\left(\mathrm{CDCl}_{3}\right): \delta$ $29.0\left(\mathrm{NCH}_{3}\right), 64.7(\mathrm{CH}), 66.5(\mathrm{C}) ; 107.3(\mathrm{CH}), 107.7(\mathrm{CH}), 119.1(\mathrm{CH}), 122.7(\mathrm{CH}), 126.8(\mathrm{CH}), 127.8$ $(\mathrm{CH}), 128.5(\mathrm{CH}), 129.7(\mathrm{C}), 130.2(\mathrm{C}), 130.5(\mathrm{C}), 130.6(\mathrm{C}), 132.3(\mathrm{CH}), 136.9(\mathrm{C}), 141.9(\mathrm{C}), 143.2$ (C), 154.0 (C), 196.3 (CO). C-NO2 was not observed under these experimental conditions. HRMS (EI): calcd for $\mathrm{C}_{21} \mathrm{H}_{12} \mathrm{~N}_{2} \mathrm{O}_{6}\left(\mathrm{M}^{+}\right)$389.0768, found 389.0768.

1-Methyl-3'-(3-methyl-6-nitro-2-oxo-2,3-dihydrobenzo[d]oxazol-5-yl)spiro[indoline-3,2'-oxiran]-2-one (7j) like-isomer: Beige solid; mp $190{ }^{\circ} \mathrm{C} ;{ }^{1} \mathrm{H}-\mathrm{NMR}\left(\mathrm{CDCl}_{3}\right): \delta 3.13\left(\mathrm{~s}, 3 \mathrm{H}, \mathrm{NCH}_{3}\right), 3.54\left(\mathrm{~s}, 3 \mathrm{H}, \mathrm{NCH}_{3}\right)$, 
$5.15(\mathrm{~s}, 1 \mathrm{H}, \mathrm{CH}), 6.93(\mathrm{dd}, J=7.8 \mathrm{~Hz}, J=0.7 \mathrm{~Hz}, 1 \mathrm{H}, \mathrm{CH}), 7.17(\mathrm{td}, J=7.5 \mathrm{~Hz}, J=0.7 \mathrm{~Hz}, 1 \mathrm{H}, \mathrm{CH})$, $7.32(\mathrm{dd}, J=7.3 \mathrm{~Hz}, J=0.7 \mathrm{~Hz}, 1 \mathrm{H}, \mathrm{CH}), 7.44(\mathrm{td}, J=7.3 \mathrm{~Hz}, J=1.4 \mathrm{~Hz}, 1 \mathrm{H}, \mathrm{CH}), 7.62(\mathrm{~s}, 1 \mathrm{H}, \mathrm{CH})$, $8.08(\mathrm{~s}, 1 \mathrm{H}, \mathrm{CH}) .{ }^{13} \mathrm{C}-\mathrm{NMR}\left(\mathrm{CDCl}_{3}\right): \delta 26.5\left(\mathrm{NCH}_{3}\right), 28.8\left(\mathrm{NCH}_{3}\right), 62.7(\mathrm{CH}), 65.0(\mathrm{C}), 106.8(\mathrm{CH})$, $108.9(\mathrm{CH}), 109.0(\mathrm{CH}), 122.2(\mathrm{CH}), 122.3(\mathrm{C}), 123.2(\mathrm{CH}), 128.8(\mathrm{C}), 130.8(\mathrm{CH}), 136.4(\mathrm{C}), 141.5$ (C), 141.7 (C), $144.8(\mathrm{CH}), 154.2$ (CO), 169.6 (CO). Anal. Calcld for $\mathrm{C}_{18} \mathrm{H}_{13} \mathrm{~N}_{3} \mathrm{O}_{6}$ (367.31) C, 58.86; H, 3.57 ; N, 11.44. Found: C, 58.85; H, 3.71; N, 11.31 .

1-Methyl-3'-(3-methyl-6-nitro-2-oxo-2,3-dihydrobenzo[d]oxazol-5-yl)spiro[indoline-3,2'-oxiran]-2-one (7j) unlike-isomer: Beige solid; mp $211{ }^{\circ} \mathrm{C} ;{ }^{1} \mathrm{H}-\mathrm{NMR}\left(\mathrm{CDCl}_{3}\right)$ : $\delta 3.33\left(\mathrm{~s}, 3 \mathrm{H}, \mathrm{NCH}_{3}\right), 3.59\left(\mathrm{~s}, 3 \mathrm{H}, \mathrm{NCH}_{3}\right)$, $5.18(\mathrm{~s}, 1 \mathrm{H}, \mathrm{CH}), 6.01(\mathrm{~d}, J=7.5 \mathrm{~Hz}, 1 \mathrm{H}, \mathrm{CH}), 6.71(\mathrm{t}, J=7.5 \mathrm{~Hz}, 1 \mathrm{H}, \mathrm{CH}), 6.88(\mathrm{~d}, J=7.7 \mathrm{~Hz}, 1 \mathrm{H}$, $\mathrm{CH}), 7.32(\mathrm{~d}, J=7.7 \mathrm{~Hz}, 1 \mathrm{H}, \mathrm{CH}), 7.57(\mathrm{~s}, 1 \mathrm{H}, \mathrm{CH}), 8.08(\mathrm{~s}, 1 \mathrm{H}, \mathrm{CH}) .{ }^{13} \mathrm{C}-\mathrm{NMR}\left(\mathrm{CDCl}_{3}\right): \delta 26.8$ $\left(\mathrm{NCH}_{3}\right), 29.0\left(\mathrm{NCH}_{3}\right), 62.2(\mathrm{CH}), 64.4(\mathrm{C}), 107.3(\mathrm{CH}), 107.8(\mathrm{CH}), 109.2(\mathrm{CH}), 119.8(\mathrm{C}), 122.0(\mathrm{CH})$, $122.4(\mathrm{CH}), 129.4(\mathrm{C}), 130.9(\mathrm{CH}), 137.0(\mathrm{C}), 141.8(\mathrm{C}), 141.9(\mathrm{C}), 145.6(\mathrm{C}), 154.0(\mathrm{CO}), 170.6(\mathrm{CO})$. HRMS (EI): calcd for $\mathrm{C}_{18} \mathrm{H}_{13} \mathrm{~N}_{3} \mathrm{O}_{6}\left(\mathrm{M}^{+}\right) 368.0877$, found 368.0876 .

\section{Conclusions}

In conclusion, we have investigated the reactivity of some new benzoxazolone derivatives formed via the TDAE strategy. This is the first example of the use of the TDAE strategy to generate a benzoxazolinonic anion, which cannot be formed via the standard organometallic strategy. This study brought to light a new and original reactivity and we have defined some limitations of the TDAE strategy. We show that 5-(bromomethyl)-3-methyl-6-nitrobenzo[ $d]$ oxazol-2(3H)-one (2), in addition to providing the expected alcohols 5a-i in moderate to good yields, furnished an unexpected ester $\mathbf{6}$ formed in $23 \%$ yield, particularly with the $p$-nitrobenzaldehyde. The reactions of 5-(dibromomethyl)-3-methyl-6-nitro-benzo[d]oxazol2(3H)-one (3) led to the expected oxiranes $7 \mathbf{a}-\mathbf{j}$ and mixtures of original stereoisomers $\mathbf{7} \mathbf{i}-\mathbf{j}$ in good yields. All these synthesized products are currently undergoing pharmacological evaluation.

\section{Acknowledgments}

This work was supported by the Centre National de la Recherche Scientifique. We express our thanks to V. Remusat for ${ }^{1} \mathrm{H}$ - and ${ }^{13} \mathrm{C}$-NMR spectra recording. A. R. Nadji Boukrouche thanks the Ministère de l'Enseignement Supérieur et de la Recherche for financial support.

\section{Author Contributions}

A.R.N.B., M.L., O.K., T.T. and P.V. conceived of and designed the study. A.R.N.B. and O.K. designed the experiments and interpreted the results.

\section{Conflicts of Interest}

The authors declare no conflict of interest. 


\section{References}

1. Lesieur, D.; Carato, P.; Bonte, J.-P.; Depreux, P.; Caignard, D.-H.; Millan, M.; Newman-Tancredi, A.; Renard, P.; Rettori, M.-C. Preparation of Piperazinylmethylbenzothiazolinones, -Benzoxazolinones, -Benzoxazinones, and Related Compounds as Central Nervous System Agents. EP 841330, 13 May 1998.

2. Fukaya, T.; Ishiyama, T.; Baba, S.; Masumoto, S. Identification of a novel benzoxazolone derivative as a selective, orally active $18 \mathrm{kDa}$ translocator protein (TSPO) ligand. J. Med. Chem. 2013, 56, 8191-8195.

3. Aichaoui, H.; Poupaert, J.H.; Lesieur, D.; Henichart, J.-P. Regioselectivity in the $C$-acylation of 2(3H)-benzoxazolones. Tetrahedron 1991, 47, 6649-6654.

4. Moussavi, Z.; Depreux, P.; Lesieur, D.; Cotelle, N.; Sauzieres, J.; Plancke, M.O.; Fruchart, J.C. Pharmacomodulation of 7-(2-methylenebutyryl)-2,3-dihydrobenzoxazin-[1,4]-3-one structure and normolipemic activity. Farmaco 1991, 46, 339-355.

5. Ucar, H.; van derpoorten, K.; Cacciaguerra, S.; Spampinato, S.; Stables, J.P.; Depovere, P.; Isa, M.; Masereel, B.; Delarge, J.; Poupaert, J.H. Synthesis and anticonvulsant activity of 2(3H)-benzoxazolone and 2(3H)-benzothiazolone derivatives. J. Med. Chem. 1998, 41, 1138-1145.

6. Courtois, M.; Mincheva, Z.; Andreu, F.; Rideau, M.; Viaud-Massuard, M.C. Synthesis and biological evaluation with plant cells of new fosmidomycin analogues containing a benzoxazolone or oxazolopyridinone ring. J. Enzym. Inhib. Med. Chem. 2004, 19, 559-565.

7. Raju, B.G.; Ciabatti, R.; Maffioli, S.I.; Singh, U.; Romano, G.; Micheluucci, E.; Tiseni, P.S.; Candiani, G.; Kim, B.; O’Dowd, H. Ramoplanin Derivatives Possessing Antibacterial Activity. US 0211603, 21 September 2006.

8. Jadhav, J.S.; Chatpalliwar, V.A.; Khadse, S.C.; Patil, R.R. Synthesis and screening of some new 2-(3H)-benzoxazolone derivatives for analgesic, antiinflammatory, and skeletal muscle relaxant activity. Indian J. Heterocycl. Chem. 2008, 17, 343-346.

9. Köksal, M.; Kelekci, N.G.; Mercanoglu, G.O.; Erdoğan, H. Synthesis and evaluation of analgesic, anti-inflammatory and antioxidant activities of new 6-acyl-3-alkyl-5-methyl-2(3H)-benzoxazolones. Arzneim. Forsch. 2008, 58, 398-404.

10. Mésangeau, C.; Narayanan, S.; Green, A.M.; Shaikh, J.; Kaushal, N.; Viard, E.; Xu, Y.; Fishback, J.A.; Poupaert, J.H.; Matsumoto, R.R.; et al. Conversion of a highly selective sigma-1 receptor-ligand to sigma-2 receptor preferring ligands with anticocaine activity. J. Med. Chem. 2008, 51, 1482-1486.

11. Poupaert, J.H.; Carato, P.; Colacino, E. 2(3H)-benzoxazolone and bioisosters as "privileged scaffold" in the design of pharmacological probes. Curr. Med. Chem. 2005, 12, 877-885.

12. Diouf, O.; Carato, P.; Depreux, P.; Bonte, J.P.; Caignard, D.H.; Guardiola-Lemaitre, B.; Rettori, M.C.; Belzung, C.; Lesieur, D. 5-HT1A and 5-HT2A ligands with anxiolytic and antipanic-like properties. Bioorg. Med. Chem. Lett. 1997, 7, 2579-2584.

13. Diouf, O.; Carato, P.; Lesieur, I.; Rettori, M.C.; Caignard, D.H. Synthesis and pharmacological evaluation of novel 4-(4-fluorobenzoyl)piperidine derivatives as mixed 5-HT1A/5-HT2A/D2 receptor ligands. Eur. J. Med. Chem. 1999, 34, 69-73. 
14. Carato, P.; Depreux, D.; Lesieur, D.; Millan, M.; Newman-Tancredi, A.; Rettori, M.C.; Caignard, D.H. Synthesis and binding studies on a new series of arylpiperazino benzazol-2-one and benzoxazin-3-one derivatives as selective D4 ligands. Drug Des. Discov. 2000, 17, 173-181.

15. Lesieur, D.; Delmas, E.; Yous, S.; Depreux, P.; Guillaumet, G.; Dacquet, C.; Levens, N.; Boutin, J.; Bennejean, C.; Renard, P. Preparation of Novel Heterocyclic Derivatives and Pharmaceutical Composition Containing Them as Hypoglycemic Agents. FR 2804431, 3 August 2001.

16. Lesieur, D.; Blanc-Delmas, E.; Bennejean, C.; Chavatte, P.; Guillaumet, G.; Dacquet, C.; Levens, N.; Boutin, J.; Renard, P. Preparation of Azolylalkylphenylalkylindolinones as Hypoglycemic Andhypolipidemic Agents. FR 2830012, 28 March 2003.

17. Takechi, N.; Ait-Mohand, S.; Medebielle, M.; Dolbier, W.R., Jr. Nucleophilic trifluoromethylation of acyl chlorides using the trifluoromethyl iodide/TDAE reagent. Tetrahedron Lett. 2002, 43, 4317-4319.

18. Pooput, C.; Médebielle, M.; Dolbier, W.F., Jr. A New and efficient method for the synthesis of trifluoromethylthio- and selenoethers. Org. Lett. 2004, 6, 301-303.

19. Pooput, C.; Dolbier, W.F., Jr.; Médebielle, M. Nucleophilic perfluoroalkylation of aldehydes, ketones, imines, disulfides, and diselenides. J. Org. Chem. 2006, 71, 3564-3568.

20. Giuglio-Tonolo, G.; Terme, T.; Médebielle, M.; Vanelle, P. Original reaction of $p$-nitrobenzyl chloride with aldehydes using tetrakis(dimethylamino)ethylene (TDAE). Tetrahedron Lett. 2003, 44, 6433-6435.

21. Giuglio-Tonolo, G.; Terme, T.; Médebielle, M.; Vanelle, P. Nitrobenzylation of $\alpha$-carbonyl ester derivatives using TDAE approach. Tetrahedron Lett. 2004, 45, 5121-5124.

22. Amiri-Attou, O.; Terme, T.; Vanelle, P. Functionalization of 6-nitrobenzo[1,3]dioxole with carbonyl compounds via TDAE methodology. Molecules 2005, 10, 545-551.

23. Montana, M.; Crozet, M.D.; Castera-Ducros, C.; Terme, T.; Vanelle, P. Rapid synthesis of new azaheterocyclic hydroxymalonate derivatives using TDAE approach. Heterocycles 2008, 75, 925-932.

24. Nadji-Boukrouche, A.R.; Khoumeri, O.; Terme, T.; Liacha, M.; Vanelle, P. Original TDAE reactivity in benzoxa- and benzothiazolone series. ARKIVOC 2010, 10, 358-370.

25. Montana, M.; Terme, T.; Vanelle, P. Original synthesis of oxiranes via TDAE methodology: reaction of 2-(dibromomethyl)quinoxaline with aromatic aldehydes. Tetrahedron Lett. 2005, 46, $8373-8376$.

26. Montana, M.; Terme, T.; Vanelle, P. Original synthesis of $\alpha$-chloro ketones in aza heterocyclic series using TDAE approach. Tetrahedron Lett. 2006, 47, 6573-6576.

27. Khoumeri, O.; Montana, M.; Terme, T.; Vanelle, P. First TDAE approach in quinonic series: Synthesis of new 2-substituted 1,4-dimethoxy-9,10-anthraquinones. Tetrahedron 2008, 64, 11237-11242.

28. Vanelle, P.; Maldonado, J.; Madadi, N.; Gueiffier, A.; Chapat, J.-P.; Crozet, M.P. SRN1 reactions in imidazo[1,2-a]pyridine series. Tetrahedron Lett. 1990, 31, 3013-3016.

29. Delmas, F.; Gasquet, M.; Timon-David, P.; Madadi, N.; Vanelle, P.; Vaille, A.; Maldonado, J. Synthesis and in vitro anti-protozoan activity of new 5-nitrothiophene oxime ether derivatives. Eur. J. Med. Chem. 1993, 28, 23-27. 
30. Gellis, A.; Vanelle, P.; Kaafarani, M.; Benakli, K.; Crozet, M.P. Synthesis and SRN1 reactions of nitrothiazoles. Tetrahedron 1997, 53, 5471-5484.

31. Crozet, M.D.; Botta, C.; Gasquet, M.; Curti, C.; Remusat, V.; Hutter, S.; Chapelle, O.; Azas, N.; de Méo, M.; Vanelle, P. Lowering of 5-nitroimidazole's mutagenicity: Towards optimal antiparasitic pharmacophore. Eur. J. Med. Chem. 2009, 44, 653-659.

32. Dunn, L.A.; Burgess, A.G.; Krauer, K.G.; Eckmann, L.; Vanelle, P.; Crozet, M.D.; Gillin, F.D.; Upcroft, P.; Upcroft, J.A. A new-generation 5-nitroimidazole can induce highly metronidazole-resistant Giardia lamblia in vitro. Int. J. Antimicrob. Agents 2010, 36, 37-42.

33. Khoumeri, O.; Crozet, M.D.; Terme, T.; Vanelle, P. Original TDAE application: Synthesis of 2-substituted-4,11-dimethoxy-anthra[2,3-b]furan-5,10-diones via intramolecular Buchwald reaction. Tetrahedron Lett. 2009, 50, 6372-6376.

34. Suzuki, F.; Trenbeath, S.; Gleim, R.D.; Sih, C.S. Total synthesis of anthracyclinones via intramolecular base-catalyzed cyclizations. J. Org. Chem. 1978, 43, 4159-4169.

35. Gökhan, N.; Köksal, M.; Küpeli, E.; Yeşilada, E.; Erdoğan, H. Some new Mannich bases of 5-methyl-2-benzoxazolinones with analgesic and anti-inflammatory activities. Turk. J. Chem. 2005, $29,445-454$.

36. Amiri-Attou, O.; Terme, T.; Médebielle M.; Vanelle, P. Original formation of benzyl benzoates by TDAE strategy. Tetrahedron Lett. 2008, 49, 1016-1020.

37. Juspin, T.; Laget, M.; Terme, T.; Azas, N.; Vanelle, P. TDAE assisted synthesis of new imidazo[2,1-b]thiazole derivatives as anti-infectious agents. Eur. J. Med. Chem. 2010, 45, 840-845.

38. Montana, M.; Correard, F.; Khoumeri, O.; Esteve, M.-A.; Terme, T.; Vanelle, P. Synthesis of new quinoxalines containing an oxirane ring by the TDAE strategy and in vitro evaluation in neuroblastoma cell lines. Molecules 2014, 19, 14987-14998.

Sample Availability: Samples of the compounds 1, 2, 3, 5a-j, 6 and $\mathbf{7 a}-\mathbf{j}$ are available from the authors.

(C) 2015 by the authors; licensee MDPI, Basel, Switzerland. This article is an open access article distributed under the terms and conditions of the Creative Commons Attribution license (http://creativecommons.org/licenses/by/4.0/). 